\title{
RIGID CARNOT ALGEBRAS: A CLASSIFICATION
}

\author{
A. AGRACHEV and A. MARIGO
}

\begin{abstract}
A Carnot algebra is a graded nilpotent Lie algebra $L=$ $L_{1} \oplus \cdots \oplus L_{r}$ generated by $L_{1}$. The bidimension of the Carnot algebra $L$ is the pair $\left(\operatorname{dim} L_{1}, \operatorname{dim} L\right)$. A Carnot algebra is said to be rigid if it is isomorphic to any of its small perturbations in the space of Carnot algebras of the prescribed bidimension. In this paper, we give a complete classification of rigid Carnot algebras. In addition to free nilpotent Lie algebras, there are 4 infinite series and 25 exceptional rigid algebras of 12 exceptional bidimensions.
\end{abstract}

\section{INTRODUCTION}

One main motivation to study Carnot algebras is their role as local nilpotent approximations of regular vector distributions.

Let $M$ be a $\left(C^{\infty}\right.$-) smooth $n$-dimensional manifold and let $\mathcal{F} \subset \operatorname{Vec} M$ be a set of smooth vector fields on $M$. Given $q \in M$ and an integer $l>0$, we set

$$
\Delta_{q}^{l}=\operatorname{span}\left\{\left[f_{1},\left[\ldots,\left[f_{i-1}, f_{i}\right] \ldots\right]\right](q): f_{j} \in \mathcal{F}, 1 \leq j \leq i, i \leq l\right\} \subseteq T_{q} M .
$$

Of course, $\Delta_{q}^{l} \subseteq \Delta_{q}^{m}$ for $l<m$. The set $\mathcal{F}$ is said to be bracket generating (or completely nonholonomic) at $q$ if there exists $r$ such that $\Delta_{q}^{r}=T_{q} M$. The minimum among these $r$ is said to be the degree of nonholonomy of $\mathcal{F}$ at $q$. The set $\mathcal{F}$ is called bracket generating if it is bracket generating at every point.

Definition 1. We say that $\mathcal{F} \subset \operatorname{Vec} M$ is regular at $q_{0} \in M$ if $\operatorname{dim} \Delta_{q}^{i}$ is constant in a neighborhood of $q_{0}$ for all $i>0$.

Let $\mathcal{F}$ be regular at $q_{0}$ and $\operatorname{dim} \Delta_{q_{0}}^{1}=d$. Take $f_{1}, \ldots, f_{d} \in \mathcal{F}$ such that vectors $f_{1}\left(q_{0}\right), \ldots, f_{d}\left(q_{0}\right)$ form a basis of $\Delta_{q_{0}}^{1}$. Then $f_{1}(q), \ldots, f_{d}(q)$ form a basis of $\Delta_{q}^{1}$ for any $q$ from a neighborhood of $q_{0}$. Hence for any $f \in \mathcal{F}$ there exist smooth functions $a_{1}, \ldots, a_{d}$ such that $f(q)=\sum_{i=1}^{d} a_{i}(q) f_{i}(q)$ for any $q$

2000 Mathematics Subject Classification. 58A30,58K50.

Key words and phrases. Nilpotent approximation, Carnot group. 
from the same neighborhood. It follows that

$$
\Delta_{q}^{l}=\operatorname{span}\left\{\left[f_{i_{1}},\left[\ldots, f_{i_{l}}\right] \ldots\right](q): 1 \leq i_{j} \leq d\right\}+\Delta_{q}^{l-1}, \quad l=1,2, \ldots .
$$

The regularity implies that one can choose vector fields from the collection

$$
\left\{\left[f_{i_{1}},\left[\ldots, f_{i_{l}}\right] \ldots\right](q): 1 \leq i_{j} \leq d\right\}
$$

so that the values of the selected fields at $q$ form a basis of $\Delta_{q}^{l} / \Delta_{q}^{l-1}$ for all $q$ sufficiently close to $q_{0}$. With these bases in hands we easily obtain the following well-known fact.

Lemma 1. Assume that $\mathcal{F} \subset \operatorname{Vec} M$ is regular at $q_{0}, v_{i}, v_{j} \in \operatorname{Vec} M$, $v_{i}(q) \in \Delta_{q}^{i}, v_{j}(q) \in \Delta_{q}^{j}$ for all $q$, and $v_{i}\left(q_{0}\right)=0$. Then $\left[v_{i}, v_{j}\right]\left(q_{0}\right) \in \Delta_{q_{0}}^{i+j-1}$.

It immediately follows from this lemma that the Lie brackets of the vector fields with values in $\Delta_{q}^{i}, i=1,2, \ldots$, induce the structure of a graded Lie algebra on the space $\sum_{i>0} \Delta_{q_{0}}^{i} / \Delta_{q_{0}}^{i-1}$. We denote this graded Lie algebra by $\operatorname{Lie}_{q_{0}} \mathcal{F}$. Obviously, $\operatorname{Lie}_{q_{0}} \mathcal{F}$ is generated by $\Delta_{q_{0}}^{1}$. In particular, $\operatorname{Lie}_{q_{0}} \mathcal{F}$ is a Carnot algebra.

Moreover, any Carnot algebra $L$ can be realized as $\operatorname{Lie}_{q_{0}} \mathcal{F}$ for some $\mathcal{F}$. Indeed, let $M$ be a Lie group with Lie algebra $L$ and $q_{0}$ be the unit element of this group. Then $L_{1}$ is a regular bracket generating set of left-invariant vector fields on $M$ and $L=\operatorname{Lie}_{q_{0}} L_{1}$.

We now turn to the generic case. Let $\mathcal{L}_{d}$ be the free Lie algebra with $d$ generators (all algebras in this paper are over $\mathbb{R}$ ); in other words, $\mathcal{L}_{d}$ is the Lie algebra of commutator polynomials of $d$ variables. We have $\mathcal{L}_{d}=\bigoplus_{i=1}^{\infty} \mathcal{L}_{d}^{i}$, where $\mathcal{L}_{d}^{i}$ is the space homogeneous commutator polynomials of degree $i$. Then

$$
\mathcal{L}_{d}^{(r)} \stackrel{\text { def }}{=} \bigoplus_{j=1}^{\infty} \mathcal{L}_{d}^{j} / \bigoplus_{j=r+1}^{\infty} \mathcal{L}_{d}^{j}
$$

is the free nilpotent Lie algebra of "length" $r$. We set

$$
\ell_{d}(i)=\operatorname{dim} \mathcal{L}_{d}^{i}, \quad \ell_{d}^{(r)}=\sum_{i=1}^{r} \ell_{d}(i)=\operatorname{dim} \mathcal{L}_{d}^{(r)} .
$$

The classical recursion expression of $\ell_{d}(i)$ is

$$
i \ell_{d}(i)=d^{i}-\sum_{j \mid i} j \ell_{d}(j)
$$

Any Carnot algebra of bidimension $(d, n)$ is a factor-algebra of $\mathcal{L}_{d}^{(n)}$ with respect to some graded ideal of codimension $n$. These algebras can be 
realized as follows. Any surjective linear mapping $A: \mathcal{L}_{d}^{(n)} \rightarrow \mathbb{R}^{n}$ induces a filtration of $\mathbb{R}^{n}$ by the subspaces

$$
E_{A}^{k}=\sum_{i=1}^{k} A \mathcal{L}_{d}^{i}, \quad k=1, \ldots, n .
$$

We set $\bar{A}_{k}: \mathcal{L}_{d}^{k} \rightarrow E_{A}^{k} / E_{A}^{k-1}$, the composition of $\left.A\right|_{\mathcal{L}_{d}^{k}}$ with the canonical factorization, and $\bar{A}=\bigoplus_{k=1}^{n} \bar{A}_{k}$, the induced mapping of the graded linear spaces.

Let $\mathfrak{A}(d, n) \subset \operatorname{Hom}\left(\mathcal{L}_{d}^{(n)}, \mathbb{R}^{n}\right)$ be the set of all surjective linear mappings $A: \mathcal{L}_{d}^{(n)} \rightarrow \mathbb{R}^{n}$ such that $\operatorname{ker} \bar{A}$ is an ideal of $\mathcal{L}_{d}^{(n)}$. If $A \in \mathfrak{A}(d, n)$, then $\mathcal{L}_{d}^{(n)} / \operatorname{ker} \bar{A}$ is a Carnot algebra and any Carnot algebra can be realized in this way. Of course, different ideals may provide isomorphic Carnot algebras.

Definition 2. A Carnot algebra $L$ of bidimension $(d, n)$ is said to be rigid if the set of $A \in \mathfrak{A}(d, n)$ such that $L \cong \mathcal{L}_{d}^{(n)} / \operatorname{ker} \bar{A}$ is an open subset of $\mathfrak{A}(d, n)$.

Here the symbol $\cong$ denotes the isomorphism relation for Carnot algebras.

Therefore, a Carnot algebra is rigid if it does not admit deformations: any admissible small perturbation of $A$ gives an isomorphic Carnot algebra. As a first step towards the classification of rigid cases, we describe a more general class of "generic" $A$ which characterizes Carnot algebras $\operatorname{Lie}_{q_{0}}\left\{f_{1}, \ldots, f_{d}\right\}$ for generic germs of $d$-tuples of vector fields.

Proposition 1. Let $\mathfrak{A}_{0}(d, n)$ be the set of all surjective linear mappings $A: \mathcal{L}_{d}^{(n)} \rightarrow \mathbb{R}^{n}$ such that

$$
\operatorname{ker} \bar{A}_{i}= \begin{cases}0, & \ell_{d}^{(i)}<n, \\ \mathcal{L}_{d}^{i}, & \ell_{d}^{(i)} \geq n .\end{cases}
$$

Then $\mathfrak{A}_{0}(d, n) \subset \mathfrak{A}(d, n)$ and $\mathfrak{A}_{0}(d, n)$ is an open everywhere dense subset of $\operatorname{Hom}\left(\mathcal{L}_{d}^{(n)}, \mathbb{R}^{n}\right)$.

Proof. Let $r=\min \left\{i: \ell_{d}^{(i)} \geq n\right\}$. Then $A \in \mathfrak{A}_{0}(d, n)$ if and only if $\left.A\right|_{\left.\right|_{i=1} ^{\oplus-1} \mathcal{L}_{d}^{i}}$ is an injective mapping and $\left.A\right|_{i=1} ^{r} \mathcal{L}_{d}^{i}$ is a surjective mapping. Surely, these properties hold for an open dense subset of $\operatorname{Hom}\left(\mathcal{L}_{d}^{(n)}, \mathbb{R}^{n}\right)$. Moreover, if $A \in \mathfrak{A}_{0}(d, n)$, then

$$
\operatorname{ker} \bar{A}=\left(\operatorname{ker} \bar{A}_{r}\right) \oplus A\left(\bigoplus_{i=r+1}^{n} \mathcal{L}_{d}^{i}\right) .
$$


In other words, $\operatorname{ker} \bar{A}$ is the direct sum of a linear subspace of $\mathcal{L}_{d}^{r}$ and $A\left(\bigoplus_{i=r+1}^{n} \mathcal{L}_{d}^{i}\right)$. Obviously, any such subspace is an ideal of $\mathcal{L}_{d}^{(n)}$.

Corollary 1. Any rigid Carnot algebra of bidimension $(d, n)$ is isomorphic to $\mathcal{L}_{d}^{(r)} / E$, where $r=\min \left\{i: \ell_{d}^{(i)} \geq n\right\}$ and $E$ is an $\left(\ell_{d}^{(r)}-n\right)$ dimensional subspace of $\mathcal{L}_{d}^{r}$.

We set $\bar{m}=\ell_{d}^{(r)}-n$. Then rigid Carnot algebras of bidimension $(d, n)$ are characterized by $\bar{m}$-dimensional subspaces of $\mathcal{L}_{d}^{r}$. Let $\operatorname{Gr}_{\bar{m}}\left(\mathcal{L}_{d}^{r}\right)$ be the Grassmannian of $\bar{m}$-dimensional subspaces of $\mathcal{L}_{d}^{r}$. Of course, not every $E \in \operatorname{Gr}_{\bar{m}}\left(\mathcal{L}_{d}^{r}\right)$ gives a rigid Carnot algebra. Moreover, not every bidimension admits a rigid Carnot algebra.

Definition 3. A bidimension $(d, n)$ is called rigid if there exists a rigid Carnot algebra of bidimension $(d, n)$.

Two Carnot algebras $\mathcal{L}_{d}^{(r)} / E_{i}, E_{i} \in \operatorname{Gr}_{\bar{m}}\left(\mathcal{L}_{d}^{r}\right), i=1,2$, are isomorphic if and only if there exists an automorphism of $\mathcal{L}_{d}^{(r)}$ which transforms $E_{1}$ into $E_{2}$. The automorphisms of the free nilpotent Lie algebra $\mathcal{L}_{d}^{(r)}$ are in a one-to-one correspondence with linear transformations of $\mathbb{R}^{d}=\mathcal{L}_{d}^{1}$. More precisely, the rule

$$
V^{(i)}\left[x_{1},\left[\ldots, x_{i}\right] \ldots\right] \stackrel{\text { def }}{=}\left[V x_{1},\left[\ldots, V x_{i}\right] \ldots\right], \quad x_{1}, \ldots, x_{i} \in \mathcal{L}_{d}^{1},
$$

provides a canonical extension of $V \in \mathrm{GL}\left(\mathbb{R}^{d}\right)$ to the automorphism $V^{(1)} \oplus$ $\cdots \oplus V^{(r)}$ of $\mathcal{L}_{d}^{(r)}$. In particular, we obtain a canonical action $V \mapsto V^{(r)}$ of $\mathrm{GL}\left(\mathbb{R}^{d}\right)$ on $\mathcal{L}_{d}^{r}$; Carnot Lie algebras $\mathcal{L}_{d}^{(r)} / E_{i}, i=1,2$, are isomorphic if and only if there exists $V \in \mathrm{GL}\left(\mathbb{R}^{d}\right)$ such that $V^{(r)} E_{1}=E_{2}$.

Let $\bar{\Phi}(V): \operatorname{Gr}_{\bar{m}}\left(\mathcal{L}_{d}^{r}\right) \rightarrow \operatorname{Gr}_{\bar{m}}\left(\mathcal{L}_{d}^{r}\right), V \in \mathrm{GL}\left(\mathbb{R}^{d}\right)$, be the induced action of $\mathrm{GL}\left(\mathbb{R}^{d}\right)$ on the Grassmannian so that $\bar{\Phi}(V)(E)=V^{(r)} E, E \in \operatorname{Gr}_{\bar{m}}\left(\mathcal{L}_{d}^{r}\right)$. The Carnot algebra $\mathcal{L}_{d}^{(r)} / E$ is rigid if and only if $E$ belongs to a fulldimensional orbit of the action $\bar{\Phi}$. In particular, the bidimension $(d, n)$ is rigid if and only if there exists a full-dimensional orbit of $\bar{\Phi}$. Moreover, such orbits are actually in a one-to-one correspondence with the isomorphism classes of rigid Carnot algebras. The action $\bar{\Phi}$ is algebraic. This implies the following assertion.

Corollary 2. Let $(d, n)$ be a rigid bidimension. Then the set of $E \in$ $\operatorname{Gr}_{\bar{m}}\left(\mathcal{L}_{d}^{r}\right)$ such that $\mathcal{L}_{d}^{(r)} / E$ is rigid is a Zarisski open (in particular, open dense) subset of $\mathrm{Gr}_{\bar{m}}\left(\mathcal{L}_{d}^{r}\right)$ and there is only a finite number of mutually nonisomorphic rigid Carnot algebras of the bidimension $(d, n)$.

In the next theorem we list all rigid bidimensions. It is convenient to give special names to some infinite series of bidimensions. For $d=2,3,4, \ldots$, 
the bidimensions $\left(d, \ell_{d}^{(i)}\right), i=1,2,3, \ldots$, are said to be free; the bidimension $(d, d+1)$ is called the Darboux bidimension, and the bidimension $(d,(d-1)(d+2) / 2)$ is called the dual Darboux bidimension.

Theorem 1. All free, Darboux, and dual Darboux bidimensions are rigid as well as bidimensions $(2 k-1,2 k+1),\left(2 k-1,2 k^{2}-k-2\right), k \geq 2$. Each of these bidimensions admits a unique up to an isomorphism rigid Carnot algebra. Moreover, there are 12 exceptional rigid bidimensions:

$$
\begin{gathered}
(2,4)_{1},(2,6)_{2},(2,7)_{2},(4,6)_{2},(4,7)_{2},(4,8)_{2}, \\
(5,8)_{2},(5,9)_{3},(5,11)_{3},(5,12)_{2},(6,8)_{2},(6,19)_{2},
\end{gathered}
$$

where the subscript $j$ in the expression $(d, n)_{j}$ indicates the number of isomorphism classes of rigid Carnot algebras for the given bidimension $(d, n)$. All other bidimensions are not rigid.

In the rest of the paper we will prove this theorem: in Sec. 2, we will give a necessary condition for a bidimension to be rigid. We obtain that only free bidimensions are rigid if the degree of nonholonomy $r$ is greater than 4 . The following sections are devoted to the analysis of bidimensions corresponding to $r=2,3,4$ : Sec. 3 for $r=2$, Sec. 4 for $r=3$, and Sec. 5 for $r=4$. We present a canonical basis and the multiplication table for any isomorphism class of rigid Carnot algebras. Then these multiplication tables are used in Sec. 6 to give the normal forms for all possible rigid Lie algebras of vector fields.

\section{Rigidity: A NECESSARY CONDITION}

We have the following assertion.

Proposition 2. Let $(d, n)$ be a rigid bidimension. Then

$$
d^{2}>\left(\ell_{d}(r)-\bar{m}\right) \bar{m} \text {. }
$$

Proof. It was shown in the previous section that to rigid Carnot algebras there correspond full-dimensional orbits of the action of GL( $\left.\mathbb{R}^{d}\right)$ on $\operatorname{Gr}_{\bar{m}}\left(\mathcal{L}_{d}^{r}\right)$. Let us compare the dimensions. We have

$$
\operatorname{dim} \operatorname{GL}\left(\mathbb{R}^{d}\right)=d^{2}, \quad \mathcal{L}_{d}^{r}=\mathbb{R}^{\ell_{d}(r)}, \quad \operatorname{dim} \operatorname{Gr}_{\bar{m}}\left(\mathcal{L}_{d}^{r}\right)=\bar{m}\left(\ell_{d}(r)-\bar{m}\right) .
$$

Taking into account that scalar multiples of the identity matrix from $\mathrm{GL}\left(\mathbb{R}^{d}\right)$ act trivially on the Grassmannian, we obtain that a necessary condition for the existence of a full-dimensional orbit is

$$
d^{2}-1 \geq\left(\ell_{d}(r)-\bar{m}\right) \bar{m}
$$

and the proposition is proved.

First, we observe that condition (1) is trivially satisfied when $\bar{m}=0$. Moreover, the condition is satisfied for some $\bar{m}$ if and only if it is satisfied for $m=\ell_{d}(r)-\bar{m}$. 
For $r=1$ we have $\ell_{d}(1)=d$ and hence, by the definition of $\bar{m}$, it must be $\bar{m}=0$. These cases correspond to the free bidimension $(d, d)$.

For $r=2$, since $\ell_{d}(2)=d(d-1) / 2$, condition (1) holds for all $\bar{m}=0,1, \ldots, \ell_{d}(2)-1$ if $d \leq 4$, for $\bar{m}=0,1, \ldots, \ell_{d}(2)-1$, with $\bar{m} \neq 5$, if $d=5$, and for all $\bar{m}=0,1,2, \ell_{d}(2)-2, \ell_{d}(2)-1$ if $d \geq 6$. Note that bidimensions corresponding to $\bar{m}=0,1, \ell_{d}(2)-1$ are free, dual Darboux, and Darboux bidimensions, respectively.

For $r=3, \ell_{d}(3)=\left(d^{3}-d\right) / 3$ and condition (1) holds for $\bar{m}=0,1, \ldots, \ell_{d}(3)-1$ if $d=2$ and for $\bar{m}=0,1, \ldots, \ell_{d}(3)-1$ if $d=3$. The bidimensions corresponding to $\bar{m}=0$ are free.

Finally, for $r=4, \ell_{d}(4)=\left(d^{4}-d^{2}\right) / 4$ and condition (1) holds for all $\bar{m}=0,1, \ldots, \ell_{d}(4)-1$ if $d=2$.

For $r>4$, condition (1) is never satisfied for $\bar{m}>0$.

In synthesis, in addition to the free bidimensions, we have the following cases to analyze:

\begin{tabular}{|l|l|c|}
\hline$r=2$ & $d=3$ & $\bar{m}=1,2$ \\
\hline & $d=4$ & $\bar{m}=1,2,3,4,5$ \\
\hline & $d=5$ & $\bar{m}=1,2,3,4,6,7,8,9$ \\
\hline & $d \geq 6$ & $\bar{m}=1,2, \ell_{d}(2)-2, \ell_{d}(2)-1$ \\
\hline$r=3$ & $d=2$ & $\bar{m}=1$ \\
\hline & $d=3$ & $\bar{m}=1,7$ \\
\hline$r=4$ & $d=2$ & $\bar{m}=1,2$ \\
\hline
\end{tabular}

Let $m=\ell_{d}(r)-\bar{m}$, and let $\mathcal{L}_{d}^{r^{\star}}$ be the adjoint space to $\mathcal{L}_{d}^{r}$. The involution $E \mapsto E^{\perp}$ sends $\bar{m}$-dimensional subspaces of $\mathcal{L}_{d}^{r}$ into $m$-dimensional subspaces of $\mathcal{L}_{d}^{r^{\star}}$. Denote by $\Phi$ the corresponding action of $\operatorname{GL}\left(\mathbb{R}^{d}\right)$ on $\operatorname{Gr}_{m}\left(\mathcal{L}_{d}^{r^{\star}}\right)$; it acts according to the rule $\Phi(V)\left(E^{\perp}\right)=(\bar{\Phi}(V) E)^{\perp}$.

In the following sections, we deal with the action $\Phi$ on $\operatorname{Gr}_{m}\left(\mathcal{L}_{d}^{r^{\star}}\right)$ rather than with the action $\bar{\Phi}$ on $\operatorname{Gr}_{\bar{m}}\left(\mathcal{L}_{d}^{r}\right)$; this makes shorter the way from the classification of subspaces to the tables of products of the Lie algebras. Moreover, we mainly work in a fixed Hall basis of $\mathcal{L}_{d}^{r}$ and do not make difference between $\mathcal{L}_{d}^{r}$ and $\mathcal{L}_{d}^{r^{\star}}$.

\section{The CASES WHERE $r=2$}

The following proposition allows us to reduce the analysis of possible rigid bidimensions for $r=2$.

Proposition 3. If $r=2$, then the bidimension $\left(d, \ell_{d}^{r-1}+m\right)$ is rigid if and only if the dual bidimension $\left(d, \ell_{d}^{r}-m\right)$ is rigid. Moreover, the number of isomorphism classes of rigid algebras for the bi-dimension $\left(d, \ell_{d}^{r-1}+m\right)$ and the dual bidimension $\left(d, \ell_{d}^{r}-m\right)$ is the same. 
Proof. Let $\Phi$ be the action on $\operatorname{Gr}_{m}\left(\mathcal{L}_{d}^{2}\right)$ as in Sec. 1. Fix $E \in \operatorname{Gr}_{m}\left(\mathcal{L}_{d}^{2}\right)$ and consider the following mappings:

$$
\begin{aligned}
\Psi: \operatorname{GL}\left(\mathbb{R}^{d}\right) \rightarrow \operatorname{Gr}_{m}\left(\mathcal{L}_{d}^{2}\right), & & V \mapsto \Phi(V)(E), \\
\tilde{\Psi}: \operatorname{GL}\left(\mathbb{R}^{d}\right) \rightarrow \operatorname{Gr}_{l_{d}(2)-m}\left(\mathcal{L}_{d}^{2}\right), & & V \mapsto \Phi(V)\left(E^{\perp}\right) .
\end{aligned}
$$

Let $V \in \mathrm{GL}\left(\mathbb{R}^{d}\right)$. Introduce the notation $V^{-T}=\left(V^{T}\right)^{-1}$. We show that for all $V \in \mathrm{GL}\left(\mathbb{R}^{d}\right), \tilde{\Psi}\left(V^{-T}\right)=(\Psi(V))^{\perp}$, so that a one-to-one correspondence between the image of $\Psi$ and that of $\tilde{\Psi}$ is established and the proposition is proved.

Let $V \in \mathrm{GL}\left(\mathbb{R}^{d}\right), V=\left(v_{i l}\right)_{i, l=1}^{d}$, and $g_{i}(q)=\sum_{l=1}^{d} v_{i l}(q) f_{l}(q), i=1, \ldots, d$. Then

$$
\left[g_{i}, g_{j}\right]=\Phi(V)\left[f_{i}, f_{j}\right]=\sum_{l<k} \operatorname{det}\left(\left[\begin{array}{cc}
v_{i l} & v_{i k} \\
v_{j l} & v_{j k}
\end{array}\right]\right)\left[f_{l}, f_{k}\right]
$$

Hence

$$
\Phi\left(V^{T}\right)=(\Phi(V))^{T}, \quad \Phi\left(V_{1} V_{2}\right)=\Phi\left(V_{1}\right) \Phi\left(V_{2}\right)
$$

for all $V, V_{1}, V_{2} \in \mathrm{GL}\left(\mathbb{R}^{d}\right)$. It follows that $\Phi\left(V^{-T}\right)=(\Phi(V))^{-T}$ and for all $w_{1} \in E$ and $w_{2} \in E^{\perp}$, we have

$$
\left\langle\Phi\left(V^{-T}\right) w_{2}, \Phi(V) w_{1}\right\rangle=w_{2}^{T}(\Phi(V))^{-1} \Phi(V) w_{1}=\left\langle w_{2}, w_{1}\right\rangle=0,
$$

which proves that $\tilde{\Psi}\left(V^{-T}\right)=(\Psi(V))^{\perp}$.

Assume that we know a multiplication table for some $m$. Then we obtain the dual multiplication table as follows.

Let $f^{\pi_{i}}, i=1, \ldots, \ell_{d}(2)$, be Lie brackets of order 2 which are linearly independent with respect to the Jacobi identity. Assume that the multiplication table gives $f^{\pi_{i}}=\sum_{j=1}^{m} \lambda_{i j} f^{\pi_{j}}$ for $i=m+1, \ldots, \ell_{d}(2)$, i.e., $\Lambda f=0$, where $\Lambda=\left[\hat{\Lambda} \mid-I_{\ell_{d}(2)-m}\right]$,

$$
\hat{\Lambda}=\left[\begin{array}{ccc}
\lambda_{(m+1) 1} & \cdots & \lambda_{(m+1) m} \\
\vdots & \ddots & \vdots \\
\lambda_{\ell_{d}(2) 1} & \cdots & \lambda_{\ell_{d}(2) m}
\end{array}\right]
$$

and $f=\left[f^{\pi_{1}}, \ldots, f^{\pi_{\ell_{d}(2)}}\right]$. Then $\Lambda^{\perp}=\left[I_{m} \mid \hat{\Lambda}^{T}\right] f$ represents the orthogonal space to the space generated by $\Lambda$ and the dual multiplication table is given by $\Lambda^{\perp} f$, i.e., $f^{\pi_{j}}=-\sum_{i=m+1}^{\ell_{d}(2)} \lambda_{i j} f^{\pi_{i}}$ for $j=1, \ldots, m$. As an example, in this paper we will give the dual multiplication table and the corresponding normal form for $m=1$. The other dual cases can be obtained similarly. 
The space $\mathcal{L}_{d}^{2}$ is identified with the wedge square $\bigwedge^{2} \mathbb{R}^{d}$. Hence any $E \in \operatorname{Gr}_{m}\left(\mathcal{L}_{d}^{2}\right)$ is identified with an $m$-dimensional vector space of antisymmetric $(d \times d)$-matrices. In order to fix the notation, we next describe this identification (and the corresponding action of $\mathrm{GL}\left(\mathbb{R}^{d}\right)$ ) in more detail.

Fix generators $f_{1}, \ldots, f_{d}$ for $\mathcal{L}_{d}^{1}$ and let $f^{\pi_{1}}, \ldots, f^{\pi_{m}}$ be such that they form a basis of $E \in \operatorname{Gr}_{m}\left(\mathcal{L}_{d}^{2}\right)$. Then we can write

$$
\left[f_{l}, f_{k}\right]=\sum_{h=1}^{m} \omega_{l k}^{h} f^{\pi_{h}} .
$$

Note that, since $\left[f_{l}, f_{k}\right]=-\left[f_{k}, f_{l}\right]$ for all $h=1, \ldots, m, \omega^{h}=\left\{\omega_{l k}^{h}\right\}_{l k}$ is an antisymmetric $(d \times d)$-matrix. For a different choice of the set $\left\{f^{\pi_{h}}, h=1, \ldots, m\right\}$, i.e.,

$$
f^{\pi_{h}}=\sum_{i=1}^{m} x_{h i} \tilde{f}^{\pi_{i}}
$$

we have

$$
\left[f_{l}, f_{k}\right]=\sum_{i=1}^{m} \tilde{\omega}_{l k}^{i} \tilde{f}^{\pi_{i}}
$$

where

$$
\tilde{\omega}^{i}=\sum_{h=1}^{m} x_{h i} \omega^{h} .
$$

Consider the space generated by $\omega^{h}, h=1, \ldots, m$, and write each element of the space under consideration as $\omega(x)=\sum_{i} x_{i} \omega^{i}, x=\left(x_{1}, \ldots, x_{m}\right)$. Let $V \in \mathrm{GL}\left(\mathbb{R}^{d}\right)$. Then

$\Phi(V)\left[f_{i}, f_{j}\right]=\sum_{l k} v_{i l} v_{j k}\left[f_{l}, f_{k}\right]=\sum_{h} \sum_{l k} v_{i l} v_{j k} \omega_{l k}^{h} f^{\pi_{h}}=\sum_{h}\left(V \omega^{h} V^{T}\right)_{i j} f^{\pi_{h}}$.

Hence $\Phi(V) \omega(x)=V \omega(x) V^{T}$.

Next we analyze all possible rigid bidimension for $r=2$ up to duality:

$(d, d+1)$ : for any $d$, corresponding to $m=1$, Sec. 3.1 ;

$(d, d+2)$ : for $d \geq 4$, corresponding to $m=2$, Sec. 3.2 ;

$(d, d+3)$ : for $d=4$ and $d=5$ corresponding to $m=3$, Sec. 3.3 ;

$(d, d+4)$ : for $d=5$, corresponding to $m=4$, Sec. 3.4 .

3.1. The case $m=1$. In this case, we have a one-dimensional space of antisymmetric $(d \times d)$-matrices. A generic antisymmetric $(d \times d)$-matrix $\omega$ can be written in the form $\omega=V D V^{T}$, where $V$ is nonsingular and $D$ is a block-diagonal matrix with blocks $D_{i}$ as described next.

- If $\omega$ is a $(d \times d)$-matrix with even $d$, then $D_{i}, i=1, \ldots, d / 2$, are all $(2 \times 2)$-matrices of the form $\left[\begin{array}{cc}0 & 1 \\ -1 & 0\end{array}\right]$. 
- If $\omega$ is a $(d \times d)$-matrix with odd $d$, then $D_{i}, i=1, \ldots(d-1) / 2$, are all $(2 \times 2)$-matrices of the form $\left[\begin{array}{cc}0 & 1 \\ -1 & 0\end{array}\right]$ and $D_{i}$ for $i=(d-1) / 2+1$ is the zero one-dimensional block.

Then we have the following assertion.

Proposition 4. The Darboux bidimension $(d, d+1)$ is rigid with a unique isomorphism class. The representing family $\mathcal{F}$ is completely described by the following multiplication table for even $d$ (for odd $d)$ :

$$
\left[f_{2 i-1}, f_{j}\right]=\left\{\begin{array}{ll}
{\left[f_{1}, f_{2}\right]} & \text { if } j=2 i, \\
0 & \text { otherwise, }
\end{array} \quad i=1, \ldots, \frac{d}{2}\left(\frac{d-1}{2} \text { for odd } d\right) .\right.
$$

By duality, also the dual Darboux bidimension $(d,(d-1)(d+1) / 2)$ is rigid with a unique isomorphism class. The multiplication table is as follows:

$$
\left[f_{1}, f_{2}\right]= \begin{cases}\sum_{i=2}^{d / 2}\left[f_{2 i-1}, f_{2 i}\right] & \text { if } d \text { is even } \\ \sum_{i=2}^{d-1) / 2}\left[f_{2 i-1}, f_{2 i}\right] & \text { if } d \text { is odd. }\end{cases}
$$

The normal forms are given in Sec. 6 (see Eqs. (32) and (33) for the Darboux and dual Darboux bidimension, respectively).

3.2. The case $m=2$. If $m=2$, then each $E \in \operatorname{Gr}_{m}\left(\mathcal{L}_{d}^{2}\right)$ is identified with a two-dimensional subspace of the vector space $\bigwedge^{2} \mathbb{R}^{d}$ of antisymmetric $(d \times d)$-matrices. We distinguish between $d$ even and odd.

Assume first that $d$ is even and that $\operatorname{Pf}(\omega)$ is the Pfaffian of the antisymmetric $(d \times d)$-matrix $\omega$. Recall that $\mathrm{Pf}$ is a degree- $d / 2$ homogeneous polynomial such that $(\operatorname{Pf}(\omega))^{2}=\operatorname{det}(\omega)$.

Let $\omega^{1}, \omega^{2} \in \bigwedge^{2} \mathbb{R}^{d}$ form a basis of the subspace $E$ under consideration, so that any element of the subspace can be written as $\omega\left(x_{1}, x_{2}\right)=x_{1} \omega^{1}+x_{2} \omega^{2}$. Consider the polynomial $p\left(x_{1}, x_{2}\right)=\operatorname{Pf}\left(\omega\left(x_{1}, x_{2}\right)\right)$. A change of the basis of $E$ induces a linear change of variables of the polynomial $p\left(x_{1}, x_{2}\right)$ and the transformation $\omega \mapsto V^{T} \omega V, V \in \mathrm{GL}\left(\mathbb{R}^{d}\right)$ preserves $p\left(x_{1}, x_{2}\right)$ up to a scalar multiplier since $\operatorname{Pf}\left(V^{T} \omega\left(x_{1}, x_{2}\right) V\right)=\operatorname{det} V \operatorname{Pf}\left(\omega\left(x_{1}, x_{2}\right)\right)$.

The following proposition holds.

Proposition 5. If $d$ is even, then the codimension of any orbit of the action $\Phi$ in $\operatorname{Gr}_{2}\left(\mathcal{L}_{d}^{2}\right)$ is no less than $d / 2-3$.

Proof. The space of degree- $d / 2$ homogeneous polynomials of two variables has dimension $d / 2+1$ and the group GL(2) of linear changes of variables in the plane is four-dimensional. The polynomials $p\left(x_{1}, x_{2}\right)=\operatorname{Pf}\left(\omega\left(x_{1}, x_{2}\right)\right)$ are invariant under the action $\Phi$ up to linear changes of variables. We have $d / 2+1-4=d / 2-3$. It remains to show that any polynomial of degree 
$d / 2$ is realized as $\operatorname{Pf}\left(\omega\left(x_{1}, x_{2}\right)\right)$. This is easy. Consider, for example,

$$
\omega^{1}=\alpha_{0}\left[\begin{array}{cccc}
J & 0 & \cdots & 0 \\
0 & J & \cdots & 0 \\
\vdots & \vdots & \ddots & \vdots \\
0 & 0 & \cdots & J
\end{array}\right], \quad \omega^{2}=\left[\begin{array}{cccc}
\alpha_{1} J & 0 & \cdots & 0 \\
0 & \alpha_{2} J & \cdots & 0 \\
\vdots & \vdots & \ddots & \vdots \\
0 & 0 & \cdots & \alpha_{\frac{d}{2}} J
\end{array}\right],
$$

where $J$ denotes the antisymmetric $(2 \times 2)$-matrix $\left[\begin{array}{cc}0 & 1 \\ -1 & 0\end{array}\right]$.

We have that

$$
\operatorname{Pf}\left(\omega\left(x_{1}, x_{2}\right)\right)=\left(\alpha_{0} x_{1}+\alpha_{1} x_{2}\right)\left(\alpha_{0} x_{1}+\alpha_{2} x_{2}\right) \cdots\left(\alpha_{0} x_{1}+\alpha_{d / 2} x_{2}\right) ;
$$

hence any polynomial of degree $d / 2$ in the variables $x_{1}$ and $x_{2}$ can be obtained by a suitable choice of $\alpha_{0}, \alpha_{1}, \alpha_{2}, \ldots, \alpha_{d / 2}$.

Corollary 3. Let $d$ be even and $(d, d+2)$ be rigid. Then $d<8$.

By Corollary 3 , we should analyze only the cases, where $d<8$, i.e., $d=4$ and $d=6$. For these cases, we have the following assertion.

Proposition 6. For $d=4$ and $d=6$, the bidimension $(d, d+2)$ is rigid with two isomorphism classes distinguished by the sign of the discriminant of the polynomial $\operatorname{Pf}\left(\omega\left(x_{1}, x_{2}\right)\right)$.

Proof. Note that the roots of

$$
\operatorname{Pf}\left(\omega\left(x_{1}, x_{2}\right)\right)=0
$$

can be:

for $d=4$ : either real or complex conjugate;

for $d=6$ : either three real or one real and two complex conjugate.

Next we provide the multiplication table for a representing family $\mathcal{F}$ for each of the above cases. This will show that the bidimensions $(d, d+2)$ are rigid and the isomorphism class is uniquely reconstructed from the number of real roots of Eq. (5).

$d=4$, real case. Consider a generic two-dimensional subspace of $\bigwedge^{2} \mathbb{R}^{d}$. Then Eq. (5) has simple roots and to form a basis of the subspace under the consideration, we can choose two corank-2, antisymmetric $(4 \times 4)$-matrices $\omega^{1}$ and $\omega^{2}$ with transversal kernels. Let $e_{1}, e_{2}, e_{3}, e_{4} \in \mathbb{R}^{4}$ be linearly independent and such that $e_{1}, e_{2} \in \operatorname{ker} \omega^{2}$ with $e_{1}^{T} \omega^{1} e_{2}=1$ and $e_{3}, e_{4} \in \operatorname{ker} \omega^{1}$ with $e_{3}^{T} \omega^{2} e_{4}=1$. By writing Eq. (2) in these coordinates, we obtain the following multiplication table:

$$
\left[f_{1}, f_{2}\right]=f^{\pi_{1}}, \quad\left[f_{3}, f_{4}\right]=f^{\pi_{2}}, \quad\left[f_{i}, f_{j}\right]=0 \text { otherwise. }
$$

The normal form for $\mathcal{F}$ is given in Sec. 6 (see Eq. (34)). 
$d=4$, complex case. Let $\left(x_{1}, 1\right),\left(\bar{x}_{1}, 1\right)$ be a pair of conjugate complex solutions of Eq. (5). Then $\omega^{1}=x_{1} \tilde{\omega}^{1}+\tilde{\omega}^{2}$ and $\omega^{2}=\bar{x}_{1} \tilde{\omega}^{1}+\tilde{\omega}^{2}$ are two corank-2, antisymmetric $(4 \times 4)$-matrices with complex coefficients such that

$$
\omega^{1}+\omega^{2}=2 \operatorname{Re}\left(\omega^{1}\right), \quad \omega^{1}-\omega^{2}=2 \imath \operatorname{Im}\left(\omega^{1}\right) .
$$

Note that it suffices to find a normal form for $\operatorname{Re}\left(\omega^{1}\right)$ and $\operatorname{Im}\left(\omega^{1}\right)$. Indeed,

$$
\begin{gathered}
\omega^{1} f^{\pi_{1}}+\omega^{2} f^{\pi_{2}}=\left(\operatorname{Re}\left(\omega^{1}\right)+\imath \operatorname{Im}\left(\omega^{1}\right)\right) f^{\pi_{1}}+\left(\operatorname{Re}\left(\omega^{1}\right)-\imath \operatorname{Im}\left(\omega^{1}\right)\right) f^{\pi_{2}} \\
=\left(\operatorname{Re}\left(\omega^{1}\right)+\imath \operatorname{Im}\left(\omega^{1}\right)\right)\left(\frac{1+\imath}{4} \tilde{f}^{\pi_{1}}+\frac{1-\imath}{4} \tilde{f}^{\pi_{2}}\right) \\
+\left(\operatorname{Re}\left(\omega^{1}\right)-\imath \operatorname{Im}\left(\omega^{1}\right)\right)\left(\frac{1-\imath}{4} \tilde{f}^{\pi_{1}}+\frac{1+\imath}{4} \tilde{f}^{\pi_{2}}\right) \\
=\left(\operatorname{Re}\left(\omega^{1}\right)+\imath \operatorname{Im}\left(\omega^{1}\right)\right)\left(\frac{1}{4}\left(\tilde{f}^{\pi_{1}}+\tilde{f}^{\pi_{2}}\right)+\frac{1}{4} \imath\left(\tilde{f}^{\pi_{1}}-\tilde{f}^{\pi_{2}}\right)\right) \\
+\left(\operatorname{Re}\left(\omega^{1}\right)-\imath \operatorname{Im}\left(\omega^{1}\right)\right)\left(\frac{1}{4}\left(\tilde{f}^{\pi_{1}}+\tilde{f}^{\pi_{2}}\right)-\frac{1}{4} \imath\left(\tilde{f}^{\pi_{1}}-\tilde{f}^{\pi_{2}}\right)\right) \\
=\frac{1}{2}\left(\operatorname{Re}\left(\omega^{1}\right)\left(\tilde{f}^{\pi_{1}}+\tilde{f}^{\pi_{2}}\right)-\operatorname{Im}\left(\omega^{1}\right)\left(\tilde{f}^{\pi_{1}}-\tilde{f}^{\pi_{2}}\right)\right) \\
=\frac{1}{2}\left(\operatorname{Re}\left(\omega^{1}\right)-\operatorname{Im}\left(\omega^{1}\right)\right) \tilde{f}^{\pi_{1}}+\frac{1}{2}\left(\operatorname{Re}\left(\omega^{1}\right)+\operatorname{Im}\left(\omega^{1}\right)\right) \tilde{f}^{\pi_{2}} .
\end{gathered}
$$

Let $p=p_{1}+\imath p_{2}$ and $q=p_{3}+\imath p_{4} \in \operatorname{ker} \omega^{1}$ with $p_{1} \operatorname{Re}\left(\omega^{1}\right) p_{3}=1$ and $p_{2} \operatorname{Im}\left(\omega^{1}\right) p_{4}=1$. Then we can write in the coordinates $p_{1}, p_{2}, p_{3}, p_{4}$ :

$$
\operatorname{Re}\left(\omega^{1}\right)=\left[\begin{array}{cccc}
0 & 0 & 1 & 1 \\
0 & 0 & 1 & -1 \\
-1 & -1 & 0 & 0 \\
-1 & 1 & 0 & 0
\end{array}\right], \quad \operatorname{Im}\left(\omega^{1}\right)=\left[\begin{array}{cccc}
0 & 0 & -1 & 1 \\
0 & 0 & 1 & 1 \\
1 & -1 & 0 & 0 \\
-1 & -1 & 0 & 0
\end{array}\right] .
$$

Finally, writing the equation $\omega=\omega^{1} \tilde{f}^{\pi_{1}}+\omega^{2} \tilde{f}^{\pi_{2}}$ in the new coordinates, we obtain the following multiplication table:

$$
\begin{gathered}
{\left[f_{1}, f_{2}\right]=\left[f_{3}, f_{4}\right]=0,} \\
{\left[f_{1}, f_{3}\right]=-\left[f_{2}, f_{4}\right]=\tilde{f}^{\pi_{1}},} \\
{\left[f_{1}, f_{4}\right]=\left[f_{2}, f_{3}\right]=\tilde{f}^{\pi_{2}} .}
\end{gathered}
$$

The normal form for $\mathcal{F}$ is given in Sec. 6 (see Eq. (35)).

$d=6$, real case. Consider a generic two-dimensional subspace of $\bigwedge^{2} \mathbb{R}^{d}$. Then Eq. (5) has simple roots $\left(x_{1}, 1\right),\left(x_{2}, 1\right)$, and $\left(x_{3}, 1\right)$. Let $\lambda_{1}, \lambda_{2}, \lambda_{3} \neq 0$ be such that $\lambda_{1}\left(x_{1}, 1\right)+\lambda_{2}\left(x_{2}, 1\right)=\lambda_{3}\left(x_{3}, 1\right)$. Then $\omega^{i}=\lambda_{i} x_{i} \tilde{\omega}^{1}+\lambda_{i} \tilde{\omega}^{2}$ for $i=1,2,3$ are antisymmetric $(6 \times 6)$-matrices such that $\omega^{3}=\omega^{1}+\omega^{2}$. Moreover, by generic assumptions, we also have that the kernels of the above matrices are transversal. Then let $p_{i} \in \mathbb{R}^{6}, i=1, \ldots, 6$, be linearly 
independent, with $p_{1}, p_{2} \in \operatorname{ker} \omega^{2}, p_{3}, p_{4} \in \operatorname{ker} \omega^{1}$, and $p_{5}, p_{6} \in \operatorname{ker} \omega^{3}$ such that $p_{1} \omega^{1} p_{2}=1$ and $p_{3} \omega^{2} p_{4}=1$. In these coordinates we can write

$$
\omega^{1}=\left[\begin{array}{ccc}
J & 0 & 0 \\
0 & 0 & 0 \\
0 & 0 & -J
\end{array}\right], \quad \omega^{2}=\left[\begin{array}{ccc}
0 & 0 & 0 \\
0 & J & 0 \\
0 & 0 & J
\end{array}\right], \quad \omega^{3}=\left[\begin{array}{ccc}
J & 0 & 0 \\
0 & J & 0 \\
0 & 0 & 0
\end{array}\right] .
$$

Finally, Eq. (2) gives the following multiplication table for $\mathcal{F}$ :

$$
\left[f_{5}, f_{6}\right]=-\left[f_{1}, f_{2}\right]+\left[f_{3}, f_{4}\right], \quad\left[f_{i}, f_{j}\right]=0 \text { otherwise. }
$$

The normal form is given in Sec. 6 (see Eq. (37)).

$d=6$, complex case. Let $\left(x_{1}, 1\right),\left(x_{2}, 1\right)$, and $\left(x_{3}, 1\right)$ be the three solutions of Eq. (5), where $x_{3} \in \mathbb{R}$ and $x_{2}=\bar{x}_{1}$ (we denote by $\bar{x}$ the conjugate of $x)$. There exist $\lambda_{1}, \lambda_{2}, \lambda_{3} \in \mathbb{C}$, where $\lambda_{2}=\bar{\lambda}_{1}$ and $\lambda_{3} \in \mathbb{R}$, such that $\omega^{i}=\lambda_{i} x_{i} \tilde{\omega}^{1}+\lambda_{i} \tilde{\omega}^{2}, i=1,2,3$, are antisymmetric with

$$
\omega^{1}+\omega^{2}=2 \operatorname{Re}\left(\omega^{1}\right), \quad \omega^{1}-\omega^{2}=2 \imath \operatorname{Im}\left(\omega^{1}\right), \quad \omega^{3}=\omega^{1}+\omega^{2}=2 \operatorname{Re}\left(\omega^{1}\right) .
$$

Let $p_{1}, p_{2} \in \operatorname{ker}\left(\omega^{3}\right)$, where $p_{1} \operatorname{Im}\left(\omega^{1}\right) p_{2}=1$ and $p_{3}, \ldots, p_{6}$ are orthogonal to the two-dimensional space generated by $\left\{\operatorname{Im}\left(\omega^{1}\right) p_{i}, i=1,2\right\}$. In these coordinates we write:

$$
\operatorname{Re}\left(\omega^{1}\right)=\left[\begin{array}{cc}
0 & 0 \\
0 & \operatorname{Re}\left(\omega^{1}\right)_{22}
\end{array}\right], \quad \operatorname{Im}\left(\omega^{1}\right)=\left[\begin{array}{cc}
J & 0 \\
0 & \operatorname{Im}\left(\omega^{1}\right)_{22}
\end{array}\right],
$$

where $\operatorname{Re}\left(\omega^{1}\right)_{22}$ and $\operatorname{Im}\left(\omega^{1}\right)_{22}$ are antisymmetric $(4 \times 4)$-matrices with $\operatorname{Re}\left(\omega^{1}\right)_{22} \pm \imath \operatorname{Im}\left(\omega^{1}\right)_{22}$ of corank 2 . Therefore, it remains to consider the complex case for $d=4$ and, with the same arguments, we can write

$$
\begin{aligned}
\operatorname{Re}\left(\omega^{1}\right)= & {\left[\begin{array}{rrrrrr}
0 & 0 & 0 & 0 & 0 & 0 \\
0 & 0 & 0 & 0 & 0 & 0 \\
0 & 0 & 0 & 0 & 1 & 1 \\
0 & 0 & 0 & 0 & 1 & -1 \\
0 & 0 & -1 & -1 & 0 & 0 \\
0 & 0 & -1 & 1 & 0 & 0
\end{array}\right], } \\
\operatorname{Im}\left(\omega^{1}\right)= & {\left[\begin{array}{rrrrrr}
0 & 1 & 0 & 0 & 0 & 0 \\
-1 & 0 & 0 & 0 & 0 & 0 \\
0 & 0 & 0 & 0 & -1 & 1 \\
0 & 0 & 0 & 0 & 1 & 1 \\
0 & 0 & 1 & -1 & 0 & 0 \\
0 & 0 & -1 & -1 & 0 & 0
\end{array}\right] }
\end{aligned}
$$

and

$$
\omega=\frac{1}{2}\left(\operatorname{Re}\left(\omega^{1}\right)-\operatorname{Im}\left(\omega^{1}\right)\right) \tilde{f}^{\pi_{1}}+\frac{1}{2}\left(\operatorname{Re}\left(\omega^{1}\right)+\operatorname{Im}\left(\omega^{1}\right)\right) \tilde{f}^{\pi_{2}} .
$$

Finally, we obtain the following multiplication table:

$$
\begin{aligned}
& {\left[f_{1}, f_{2}\right]=\tilde{f}^{\pi_{1}}-\tilde{f}^{\pi_{2}}, \quad\left[f_{3}, f_{5}\right]=-\left[f_{4}, f_{6}\right]=\tilde{f}^{\pi_{1}},} \\
& {\left[f_{3}, f_{6}\right]=\left[f_{4}, f_{5}\right]=\tilde{f}^{\pi_{2}}, \quad\left[f_{i}, f_{j}\right]=0 \text { otherwise. }}
\end{aligned}
$$


The normal form for $\mathcal{F}$ is given in Sec. 6 (see Eq. (38)).

To complete the analysis for $m=2$, it remains to study the cases where $d$ is odd.

Proposition 7. Let $d=2 k+1$. Then the bidimension $(d, d+2)$ is rigid with only one normal form.

This result can be deduced from the classical Kronecker's classification of singular matrix pencils (see [8] and [7]) and we omit the proof.

3.3. The case $m=3$. In this case, we deal with the three-dimensional space of antisymmetric $(d \times d)$-matrices.

Proposition 8. The bidimension $(4,7)$ is rigid with two isomorphism classes distinguished by the signature of the quadratic form

$$
\operatorname{Pf}\left(x_{1} \omega^{1}+x_{2} \omega^{2}+x_{3} \omega^{3}\right) .
$$

Proof. The equation $\operatorname{Pf}\left(x_{1} \omega^{1}+x_{2} \omega^{2}+x_{3} \omega^{3}\right)=0$ can be rewritten as follows:

$$
\left[x_{1}, x_{2}, x_{3}\right] A\left[x_{1}, x_{2}, x_{3}\right]^{T}=0,
$$

where $A$ is a symmetric $(3 \times 3)$-matrix. Depending on the signature of $A$, we either have real roots (corresponding to an indefinite matrix $A$ ) or complex roots (corresponding to sign-definite $A$ ). Next, we provide the multiplication table for a representing family $\mathcal{F}$ for each of these cases. This will show that the bidimensions $(d, d+3)$ are rigid and the isomorphism class is uniquely reconstructed from the number of real roots of Eq. (10).

$d=4$, real case. Consider a generic three-dimensional subspace of $\bigwedge^{2} \mathbb{R}^{d}$. Then the matrix $A$ of Eq. (10) is nondegenerate. If $A$ is not sign-definite, then we can assume that

$$
A=\left[\begin{array}{ccc}
0 & \frac{1}{2} & 0 \\
\frac{1}{2} & 0 & 0 \\
0 & 0 & 1
\end{array}\right]
$$

Hence the real solutions of Eq. (10) are

$$
\begin{aligned}
& {\left[x_{11}, x_{21}, x_{31}\right]=[1,0,0],} \\
& {\left[x_{12}, x_{22}, x_{32}\right]=[0,1,0],} \\
& {\left[x_{13}, x_{23}, x_{33}\right]=[a, b, c], \quad \text { where } a b+c^{2}=0 .}
\end{aligned}
$$

Then $\omega^{1}=\tilde{\omega}^{1}, \omega^{2}=\tilde{\omega}^{2}$, and $\omega^{3}=a \tilde{\omega}^{1}+b \tilde{\omega}^{2}+c \tilde{\omega}^{3}$, where $a b+c^{2}=0$, have corank 2. Moreover, under generic assumptions, we have that the kernels of $\omega^{1}, \omega^{2}$, and $\omega^{3}$ are transversal. In the coordinates $p_{1}, p_{2} \in \operatorname{ker}\left(\omega^{2}\right)$, 
$p_{3}, p_{4} \in \operatorname{ker}\left(\omega^{1}\right)$, with $p_{1} \omega^{1} p_{2}=1$ and $p_{3} \omega^{2} p_{4}=1$, we write:

$$
\omega^{1}=\left[\begin{array}{rrrr}
0 & 1 & 0 & 0 \\
-1 & 0 & 0 & 0 \\
0 & 0 & 0 & 0 \\
0 & 0 & 0 & 0
\end{array}\right], \quad \omega^{2}=\left[\begin{array}{rrrr}
0 & 0 & 0 & 0 \\
0 & 0 & 0 & 0 \\
0 & 0 & 0 & 1 \\
0 & 0 & -1 & 0
\end{array}\right]
$$

and

$$
\begin{aligned}
\omega^{3} & =a \tilde{\omega}^{1}+b \tilde{\omega}^{2}+c \tilde{\omega}^{3} \\
& =\left[\begin{array}{cccc}
0 & a+c w_{12} & c w_{13} & c w_{14} \\
-a-c w_{12} & 0 & c w_{23} & c w_{24} \\
-c w_{13} & -c w_{23} & 0 & b+c w_{34} \\
-c w_{14} & -c w_{24} & -b-c w_{34} & 0
\end{array}\right],
\end{aligned}
$$

where $w_{i j}$ are the components of $\tilde{\omega}^{3}$. Since $\omega^{3}$ has corank 2, the following condition holds for all $a, b$, and $c$ such that $a b+c^{2}=0$ :

$$
0=\left(a+c w_{12}\right)\left(b+c w_{34}\right)-c^{2}\left(w_{13} w_{24}-w_{23} w_{14}\right) .
$$

By Eq. (11), it follows that $w_{12}=w_{34}=0$ and

$$
w_{13} w_{24}-w_{23} w_{14}=-1 .
$$

In particular, setting $a=1, b=-1$, and $c=1$, we obtain that $\omega^{3}$ has the form

$$
\omega^{3}=\left[\begin{array}{cc}
J & \left(\tilde{\omega}^{3}\right)_{12} \\
-\left(\tilde{\omega}^{3}\right)_{12}^{T} & -J
\end{array}\right],
$$

where by $\left(\tilde{\omega}^{3}\right)_{i j}$ we denote the $(i, j)$ th $(2 \times 2)$-block of the block matrix decomposition of $\tilde{\omega}^{3}$. Now let $P_{1}=\left(\tilde{\omega}^{3}\right)_{12}^{-T}$. Then, by Eq. (12), $\operatorname{det}\left(P_{1}\right)=-1$ and, setting

$$
P=\left[\begin{array}{cc}
P_{1} & 0 \\
0 & I_{2}
\end{array}\right]
$$

we obtain

$$
\begin{gathered}
(P)^{T} \omega^{1} P=\left[\begin{array}{cc}
-J & 0 \\
0 & 0
\end{array}\right], \quad(P)^{T} \omega^{2} P=\left[\begin{array}{ll}
0 & 0 \\
0 & J
\end{array}\right], \\
(P)^{T} \omega^{3} P=\left[\begin{array}{cc}
-J & I_{2} \\
-I_{2} & -J
\end{array}\right] .
\end{gathered}
$$

Finally,

$$
\omega=\left[\begin{array}{rrrr}
0 & -1 & 0 & 0 \\
1 & 0 & 0 & 0 \\
0 & 0 & 0 & 0 \\
0 & 0 & 0 & 0
\end{array}\right] f^{\pi_{1}}+\left[\begin{array}{rrrr}
0 & 0 & 0 & 0 \\
0 & 0 & 0 & 0 \\
0 & 0 & 0 & 1 \\
0 & 0 & -1 & 0
\end{array}\right] f^{\pi_{2}}
$$




$$
+\left[\begin{array}{rrrr}
0 & -1 & 1 & 0 \\
1 & 0 & 0 & 1 \\
-1 & 0 & 0 & -1 \\
0 & -1 & 1 & 0
\end{array}\right] f^{\pi_{3}}
$$

which gives the following multiplication table:

$$
\begin{aligned}
& {\left[f_{1}, f_{2}\right]=-f^{\pi_{1}}-f^{\pi_{3}},} \\
& {\left[f_{3}, f_{4}\right]=f^{\pi_{2}}-f^{\pi_{3}},} \\
& {\left[f_{1}, f_{3}\right]=\left[f_{2}, f_{4}\right]=f^{\pi_{3}},} \\
& {\left[f_{1}, f_{4}\right]=\left[f_{2}, f_{3}\right]=0 .}
\end{aligned}
$$

The normal form is given in Sec. 6 (see Eq. (40)).

$d=4$, complex case. A positive-definite matrix $A$ can be represented in the following form:

$$
A=\left[\begin{array}{lll}
1 & 0 & 0 \\
0 & 1 & 0 \\
0 & 0 & 1
\end{array}\right]
$$

Consider the complex solutions $\left[x_{11}, x_{21}, x_{31}\right]=[1, i, 0],\left[x_{12}, x_{22}, x_{32}\right]=$ $[1,-i, 0]$, and $\left[x_{13}, x_{23}, x_{33}\right]=[a, b, c]$, where $a^{2}+b^{2}+c^{2}=0$. With this choice, $\omega^{1}=\tilde{\omega}^{1}+\imath \tilde{\omega}^{2}, \omega^{2}=\tilde{\omega}^{1}-\imath \tilde{\omega}^{2}$, and $\omega^{3}=a \tilde{\omega}^{1}+b \tilde{\omega}^{2}+c \tilde{\omega}^{3}$, where $a^{2}+b^{2}+c^{2}=0$, have corank 2. Moreover, following the same arguments as for the complex case with $m=2$ and $d=4$, we can write

$$
\begin{aligned}
& \operatorname{Re}\left(\omega^{1}\right)=\tilde{\omega}^{1}=\left[\begin{array}{rrrr}
0 & 0 & 1 & 1 \\
0 & 0 & 1 & -1 \\
-1 & -1 & 0 & 0 \\
-1 & 1 & 0 & 0
\end{array}\right], \\
& \operatorname{Im}\left(\omega^{1}\right)=\tilde{\omega}^{2}=\left[\begin{array}{rrrr}
0 & 0 & -1 & 1 \\
0 & 0 & 1 & 1 \\
1 & -1 & 0 & 0 \\
-1 & -1 & 0 & 0
\end{array}\right] .
\end{aligned}
$$

In the same coordinates we write

$$
\begin{aligned}
\omega^{3} & =a \tilde{\omega}^{1}+b \tilde{\omega}^{2}+c \tilde{\omega}^{3} \\
& =\left[\begin{array}{cccc}
0 & c w_{12} & a-b+c w_{13} & a+b+c w_{14} \\
-c w_{12} & 0 & a+b+c w_{23} & -a+b+c w_{24} \\
-a+b-c w_{13} & -a-b-c w_{23} & 0 & c w_{34} \\
-a-b-c w_{14} & a+b-c w_{24} & -c w_{34} & 0
\end{array}\right],
\end{aligned}
$$

where $w_{i j}$ are the components of $\tilde{\omega}^{3}$. Since $\omega^{3}$ has corank 2, the following condition holds for all $a, b, c$ such that $a^{2}+b^{2}+c^{2}=0$ :

$$
\begin{aligned}
2\left(a^{2}+b^{2}\right)+b c\left(w_{23}\right. & \left.+w_{14}+w_{24}-w_{13}\right) \\
+ & a c\left(w_{23}+w_{14}-w_{24}+w_{13}\right)+c^{2} \sqrt{\operatorname{det}\left(\tilde{\omega}^{3}\right)}=0 .
\end{aligned}
$$


By Eq. (15), it follows that $w_{13}=w_{24}, w_{23}=-w_{14}$, and $\sqrt{\operatorname{det}\left(\tilde{\omega}_{3}\right)}=1$, hence $w_{12} w_{34}-w_{13}^{2}-w_{14}^{2}=1$. Let now $P_{1}=-\left(\tilde{\omega}^{3}\right)_{11}^{-1}\left(\tilde{\omega}^{3}\right)_{12}$, where by $\left(\tilde{\omega}^{3}\right)_{i j}$ we denote the $(i, j)$ th $(2 \times 2)$-block of the block matrix decomposition of $\tilde{\omega}^{3}$, and

Then we obtain

$$
P=\left[\begin{array}{cc}
\frac{1}{\sqrt{w_{12}}} I_{2} & \sqrt{w_{12}} P_{1} \\
0 & \sqrt{w_{12}} I_{2}
\end{array}\right]
$$

$$
(P)^{T} \tilde{\omega}^{3} P=\left[\begin{array}{ll}
J & 0 \\
0 & J
\end{array}\right]
$$

while $\tilde{\omega}_{1}$ and $\tilde{\omega}_{2}$ remain unchanged. In particular, by setting $a=0, b=\imath$, $c=1$, we have

$$
(P)^{T} \omega_{3} P=\imath(P)^{T} \tilde{\omega}_{2} P+(P)^{T} \tilde{\omega}_{3} P
$$

and

$$
\begin{gathered}
\omega=\left[\begin{array}{rrrr}
0 & 0 & 1 & 1 \\
0 & 0 & 1 & -1 \\
-1 & -1 & 0 & 0 \\
-1 & 1 & 0 & 0
\end{array}\right] f^{\pi_{1}}+\left[\begin{array}{rrrr}
0 & 0 & -1 & 1 \\
0 & 0 & 1 & 1 \\
1 & -1 & 0 & 0 \\
-1 & -1 & 0 & 0
\end{array}\right] f^{\pi_{2}} \\
+ \\
+\left[\begin{array}{rrrr}
0 & 1 & 0 & 0 \\
-1 & 0 & 0 & 0 \\
0 & 0 & 0 & 1 \\
0 & 0 & -1 & 0
\end{array}\right] f^{\pi_{3}} .
\end{gathered}
$$

The corresponding multiplication table is

$$
\begin{aligned}
& {\left[f_{1}, f_{2}\right]=\left[f_{3}, f_{4}\right]=f^{\pi_{3}},} \\
& {\left[f_{1}, f_{3}\right]=-\left[f_{2}, f_{4}\right]=f^{\pi_{1}}-f^{\pi_{2}},} \\
& {\left[f_{1}, f_{4}\right]=\left[f_{2}, f_{3}\right]=f^{\pi_{1}}+f^{\pi_{2}} .}
\end{aligned}
$$

The normal form is given in Sec. 6 (see Eq. (41)).

Now let $d=5$. Recall that an antisymmetric matrix can be considered as a skew form of degree 2 , we consider the wedge products $v^{i j}=\omega^{i} \wedge \omega^{j}$ for $i<j$ which are 4 -forms in $\mathbb{R}^{5}$. We then have that $v^{i j}, i \leq j, i, j=1,2,3$, are 6 vectors in $\mathbb{R}^{5}$. Let $\alpha_{i j} \in \mathbb{R}, i \leq j, i=1,2,3$, be such that $\sum \alpha_{i j} v^{i j}=0$. Taking $\tilde{\omega}^{i}=\sum_{h=1}^{3} x_{h i} \omega^{h}$, we have

$$
\tilde{v}_{i j}=\sum_{h k} x_{h i} x_{k j} v_{h k}, \quad \alpha_{h k}=\sum_{h k} x_{h i} x_{k j} \tilde{\alpha}_{i j} .
$$

That is the symmetric matrix $A$ of coefficients $\alpha_{i j}$,

$$
A=\left[\begin{array}{ccc}
\alpha_{11} & \frac{\alpha_{12}}{2} & \frac{\alpha_{13}}{2} \\
\frac{\alpha_{12}}{2} & \alpha_{22} & \frac{\alpha_{23}}{2} \\
\frac{\alpha_{13}}{2} & \frac{\alpha_{23}}{2} & \alpha_{33}
\end{array}\right],
$$


is mapped to $x^{-T} A x^{-1}$, where

$$
x=\left[\begin{array}{lll}
x_{11} & x_{21} & x_{31} \\
x_{12} & x_{22} & x_{32} \\
x_{13} & x_{23} & x_{33}
\end{array}\right] .
$$

The matrix $A$ is defined up to a nonzero scalar multiplier, hence the transformation $A \mapsto-A$ is also allowed. We have the following assertion.

Proposition 9. The bidimension $(5,8)$ is rigid with two isomorphism classes distinguished by the signature of the symmetric matrix $A$.

Proof. First, observe that $\omega \wedge \omega \in \operatorname{ker} \omega$ if $\operatorname{corank}(\omega)=1$ and $\omega \wedge \omega=0$ if $\operatorname{corank}(\omega)>1$. Then

$$
V^{T} \omega V\left(V^{-1} \omega \wedge \omega\right)=V^{T} \omega(\omega \wedge \omega)=0 .
$$

Now, since

$$
\left(x_{1} \omega^{1}+x_{2} \omega^{2}+x_{3} \omega^{3}\right) \wedge\left(x_{1} \omega^{1}+x_{2} \omega^{2}+x_{3} \omega^{3}\right)=\sum_{i j} x_{i} x_{j} \omega^{i} \wedge \omega^{j}=\sum_{i j} x_{i} x_{j} v_{i j},
$$

we have that, under the action of $V \in \mathrm{GL}\left(\mathbb{R}^{5}\right)$, each vector $v_{i j}$ is mapped into $V^{-1} v_{i j}$. Hence the coefficients $\alpha_{i j}$ of $A$ remain unchanged under the action of $\operatorname{GL}\left(\mathbb{R}^{5}\right)$. This fact shows that the signature of the symmetric matrix $A$ is an invariant for the bidimension $(5,8)$.

Under generic assumptions the matrix $A$ is nondegenerate and the possibly arising signatures of $A$ are +++ and ++- . Next we provide the multiplication table for a representing family $\mathcal{F}$ for each of the two cases. Thus we will show that $(5,8)$ is a rigid bidimension with two isomorphism classes.

We can assume that $A$ has the form either

$$
A=\left[\begin{array}{rrr}
1 & 0 & 0 \\
0 & 1 & 0 \\
0 & 0 & -1
\end{array}\right]
$$

or

$$
A=\left[\begin{array}{lll}
1 & 0 & 0 \\
0 & 1 & 0 \\
0 & 0 & 1
\end{array}\right],
$$

depending on the signature of $A$. Recalling the geometric meaning of the coefficients of $A$, we have $v_{11}+v_{22}=v_{33}$ or $v_{11}+v_{22}=-v_{33}$, in the first and second case, respectively. First, we choose a coordinate systems for the case $m=2$ and $d=5$. Then we can write

$$
\omega^{1}=\left[\begin{array}{cccc|c} 
& J & 0 & 0 \\
& & & & 0 \\
& 0 & 0 & & 1 \\
\hline 0 & 0 & -1 & 0 & 0
\end{array}\right], \quad \omega^{2}=\left[\begin{array}{rrrr|l}
0 & 0 & 1 \\
& & & 0 \\
0 & J & 0 \\
-1 & 0 & 0 & 0 & 0
\end{array}\right] .
$$


In these coordinates $v_{11}=[0,0,0,1,0]$ and $v_{22}=[0,1,0,0,0]$. Therefore, $v_{33}= \pm[0,1,0,1,0]$ for the first and second case. Since $\omega^{3} v_{33}=0$, we have that $\omega^{3}$ has the form

$$
\omega^{3}=\left[\begin{array}{ccccc}
0 & w_{12} & w_{13} & -w_{12} & w_{15} \\
-w_{12} & 0 & w_{23} & 0 & w_{25} \\
-w_{13} & -w_{23} & 0 & w_{23} & w_{35} \\
w_{12} & 0 & -w_{23} & 0 & -w_{25} \\
-w_{15} & -w_{25} & -w_{35} & w_{25} & 0
\end{array}\right]
$$

where $w_{12} w_{35}+w_{15} w_{23}-w_{13} w_{25}= \pm 1$. Computing now $v_{i j}$ for $i<j$, we have

$$
\begin{gathered}
v_{12}=[0,0,0,0,-1], \quad v_{13}= \pm\left[0,-w_{12},-w_{25},-w_{35}-w_{12}, w_{23}\right] \\
v_{12}= \pm\left[w_{25},-w_{25}-w_{23}, 0,-w_{23}, w_{12}\right] .
\end{gathered}
$$

Taking $P=\frac{1}{\sqrt{w_{25}}}\left[v_{11}, v_{22}, v_{12}, v_{13}, v_{23}\right]$, we obtain:

$$
P^{T} \omega^{1} P=\left[\begin{array}{rrrr|r}
0 & 0 & 0 & 0 & 0 \\
0 & 0 & 0 & 0 & 1 \\
0 & 0 & 0 & 1 & 0 \\
0 & 0 & -1 & 0 & 0 \\
\hline 0 & -1 & 0 & 0 & 0
\end{array}\right], \quad P^{T} \omega^{2} P=\left[\begin{array}{rrrr|r}
0 & 0 & 0 & -1 & 0 \\
0 & 0 & 0 & 0 & 0 \\
0 & 0 & 0 & 0 & -1 \\
1 & 0 & 0 & 0 & 0 \\
\hline 0 & 0 & 1 & 0 & 0
\end{array}\right]
$$

and, either

$$
P^{T} \omega^{3} P=\left[\begin{array}{rrrr|r}
0 & 0 & 1 & 0 & 0 \\
0 & 0 & -1 & 0 & 0 \\
-1 & 1 & 0 & 0 & 0 \\
0 & 0 & 0 & 0 & -1 \\
\hline 0 & 0 & 0 & 1 & 0
\end{array}\right]
$$

if the first case holds, or

$$
P^{T} \omega^{3} P=\left[\begin{array}{rrrr|r}
0 & 0 & 1 & 0 & 0 \\
0 & 0 & -1 & 0 & 0 \\
-1 & 1 & 0 & 0 & 0 \\
0 & 0 & 0 & 0 & 1 \\
\hline 0 & 0 & 0 & -1 & 0
\end{array}\right]
$$

if the second case holds. Finally, by

$$
\omega=\left[\begin{array}{rrrr|r}
0 & 0 & 0 & 0 & 0 \\
0 & 0 & 0 & 0 & 1 \\
0 & 0 & 0 & 1 & 0 \\
0 & 0 & -1 & 0 & 0 \\
\hline 0 & -1 & 0 & 0 & 0
\end{array}\right] f^{\pi_{1}}+\left[\begin{array}{rrrr|r}
0 & 0 & 0 & -1 & 0 \\
0 & 0 & 0 & 0 & 0 \\
0 & 0 & 0 & 0 & -1 \\
1 & 0 & 0 & 0 & 0 \\
\hline 0 & 0 & 1 & 0 & 0
\end{array}\right] f^{\pi_{2}}
$$




$$
+\left[\begin{array}{rrrr|r}
0 & 0 & 1 & 0 & 0 \\
0 & 0 & -1 & 0 & 0 \\
-1 & 1 & 0 & 0 & 0 \\
0 & 0 & 0 & 0 & \mp 1 \\
\hline 0 & 0 & 0 & \pm 1 & 0
\end{array}\right] f^{\pi_{3}}
$$

we have the following multiplication table:

$$
\begin{aligned}
& {\left[f_{2}, f_{5}\right]=\left[f_{3}, f_{4}\right]=f^{\pi_{1}}} \\
& {\left[f_{1}, f_{4}\right]=\left[f_{3}, f_{5}\right]=-f^{\pi_{2}},} \\
& {\left[f_{1}, f_{3}\right]=-\left[f_{2}, f_{3}\right]=\mp\left[f_{4}, f_{5}\right]=f^{\pi_{3}},} \\
& {\left[f_{1}, f_{2}\right]=\left[f_{1}, f_{5}\right]=\left[f_{2}, f_{4}\right]=0 .}
\end{aligned}
$$

The normal forms are given in Sec. 6 (see Eq. (42)).

3.4. The case $m=4$. Recall that for $m=4$, the only case to analyze is the case of $d=5$. A simple calculation shows that the submanifold of rank-2 antisymmetric $(5 \times 5)$-matrices has codimension 3 in the projectivized space $\bigwedge^{2} \mathbb{R}^{5}$ of all antisymmetric $(5 \times 5)$-matrices. Let $x=\left[x_{1}, x_{2}, x_{3}, x_{4}\right]$ and

$$
\omega(x)=x_{1} \omega^{1}+x_{2} \omega^{2}+x_{3} \omega^{3}+x_{4} \omega^{4}
$$

be a generic 4-dimensional vector subspace (or a three-dimensional projective subspace) of $\bigwedge^{2} \mathbb{R}^{5}$. Then $\omega(x)$ intersects the submanifold of rank-2 matrices in a finite number of points. We show that the bidimension $(5,9)$ is rigid with isomorphism classes distinguished by the number of these points which we are going to locate effectively.

First of all, we may assume without loss of generality that

$$
w=\sum_{i=1}^{4}\left(x_{i} \omega^{i}\right)_{12}
$$

does not vanish in rank-2 points. Provided that $w \neq 0$, we can assume the following block matrix decomposition (the Schur theorem):

$$
\omega(x)=\left[\begin{array}{cc}
\omega_{11}(x) & 0 \\
0 & \omega_{22}(x)
\end{array}\right],
$$

where $\omega_{11}(x)=w J$ is nonsingular antisymmetric $(2 \times 2)$-matrix and

$$
\omega_{22}(x)=\frac{1}{w}\left[\begin{array}{ccc}
0 & d_{5} & d_{4} \\
-d_{5} & 0 & d_{3} \\
-d_{4} & -d_{3} & 0
\end{array}\right]
$$

is antisymmetric $(3 \times 3)$-matrix, where $d_{i}=d_{i}(\omega(x))$ is the Pfaffian of the $i$ th principal minor of order 4 of $\omega(x)$. Then we have that $\omega(x)$ has rank 2 if and only if $d_{3}, d_{4}$, and $d_{5}$ are zero, i.e., if $x$ is the root of 3 homogeneous polynomials of degree 2 , with the additional condition that $w \neq 0$. Of 
course, $d_{1}$ and $d_{2}$ also vanish at such a root (otherwise $\omega(x)$ would have rank 4).

Proposition 10. The bidimension $(5,9)$ is rigid with three isomorphism classes distinguished by the number of real solutions of the system $d_{i}=0$, $i=1, \ldots, 5$.

Proof. As in the proof of Proposition 9, we have that under the action of $V \in \mathrm{GL}\left(\mathbb{R}^{5}\right)$, each vector $v_{i j}$ is mapped into $\tilde{v}_{i j}=V^{-1} v_{i j}$. Then

$$
\begin{aligned}
\tilde{d}_{k} & =d_{k}\left(V^{T} \omega(x) V\right)=\sum_{i j} x_{i} x_{j}\left(\tilde{v}_{i j}\right)_{k}=\sum_{i j} x_{i} x_{j}\left(\sum_{h}\left(V^{-1}\right)_{k h}\left(v_{i j}\right)_{h}\right) \\
& =\sum_{h}\left(V^{-1}\right)_{k h}\left(\sum_{i j} x_{i} x_{j}\left(v_{i j}\right)_{h}\right)=\sum_{h}\left(V^{-1}\right)_{k h} d_{k},
\end{aligned}
$$

where $(v)_{k}$ (respectively, $(V)_{k h}$ ) denotes the $k$ th (respectively, $(k h)$ th) component of the vector $v$ (respectively, of the matrix $V$ ); thus, we obtain that $\tilde{d}_{k}$ belongs to the linear space generated by $d_{1}, \ldots, d_{5}$. This shows that such a linear space is invariant under the action of $\Phi$.

Now we assume that $\omega^{1}, \omega^{2}$, and $\omega^{3}$ are in the normal form obtained for $m=3$ and $d=5$. Then $\omega(x)$ is equal to

$$
\left[\begin{array}{ccccc}
0 & x_{4} w_{12} & x_{3}+x_{4} w_{13} & -x_{2}+x_{4} w_{14} & x_{4} w_{15} \\
-x_{4} w_{12} & 0 & -x_{3}+x_{4} w_{23} & x_{4} w_{24} & x_{1}+x_{4} w_{25} \\
-x_{3}-x_{4} w_{13} & x_{3}-x_{4} w_{23} & 0 & x_{1}+x_{4} w_{34} & -x_{2}+x_{4} w_{35} \\
x_{2}-x_{4} w_{14} & -x_{4} w_{24} & -x_{1}-x_{4} w_{34} & 0 & \pm x_{3}+x_{4} w_{45} \\
-x_{4} w_{15} & -x_{1}-x_{4} w_{25} & x_{2}-x_{4} w_{35} & \mp x_{3}-x_{4} w_{45} & 0
\end{array}\right],
$$

where $w_{i j}$ are the coefficients of $\omega^{4}$. The computation of $d_{i}$ for $i=3,4,5$ yields

$$
\begin{aligned}
& d_{3}=d_{3}\left(\omega^{4}\right) x_{4}^{2}+\left(-w_{14} x_{1}+w_{25} x_{2} \pm w_{12} x_{3}\right) x_{4}+x_{1} x_{2}, \\
& d_{4}=d_{4}\left(\omega^{4}\right) x_{4}^{2}+\left(-w_{13} x_{1}-w_{12} x_{2}-\left(w_{25}+w_{15}\right) x_{3}\right) x_{4}-x_{1} x_{3}, \\
& d_{5}=d_{5}\left(\omega^{4}\right) x_{4}^{2}+\left(w_{12} x_{1}-w_{23} x_{2}-\left(w_{24}+w_{14}\right) x_{3}\right) x_{4}+x_{3} x_{2} .
\end{aligned}
$$

Generically, there are 8 solutions of the system $d_{i}=0, i=3,4,5$. Note that 3 out of 8 solutions correspond to solutions with $x_{4}=0$. Since such kind of solution violates the condition $w \neq 0$, it must be discarded. There remains 5 solutions. We can have:

(1) five real solutions;

(2) three real and two complex conjugate solutions;

(3) one real and two pairs of complex conjugate solutions.

Next we provide the multiplication table for a representing family $\mathcal{F}$ for each of these cases. This will show that $(5,9)$ is a rigid bidimension with three isomorphism classes. 
Case (1). Assume that the five solutions $x^{i}=\left[x_{1 i}, x_{2 i}, x_{3 i}, x_{4 i}\right]$, $i=1, \ldots, 5$, are all real and, since $\omega(x)$ is a generic subspace, 4 by 4 linearly independent. Let $\lambda_{1}, \ldots, \lambda_{5} \neq 0$ be such that

$$
\sum_{i=1}^{4} \lambda_{i} x^{i}=\lambda_{5} x^{5} .
$$

Let $\tilde{\omega}^{i}=\omega\left(\lambda_{i} x^{i}\right)$. By the choice of $\lambda_{i}$ we have that

$$
\sum_{i=1}^{4} \tilde{\omega}_{i}=\tilde{\omega}_{5} .
$$

Now assume that for all $i=1, \ldots, 4, \tilde{\omega}^{i}$ has rank 2 and denote by $V_{i}$ its kernel, which is a three-dimensional space, and by $v_{i j}$ the one-dimensional space $V_{i} \cap V_{j}$ (recall that, generically, $V_{i}$ are transversal). With this notation we have that

$$
\begin{array}{ll}
V_{1}=\left\{v_{12}, v_{13}, v_{14}\right\}, & V_{2}=\left\{v_{12}, v_{23}, v_{24}\right\}, \\
V_{3}=\left\{v_{13}, v_{23}, v_{34}\right\}, & V_{4}=\left\{v_{14}, v_{24}, v_{34}\right\} .
\end{array}
$$

Now let $P=\left[v_{12}, v_{13}, v_{14}, v_{23}, v_{24}\right]$, where $v_{i j}$ are suitably rescaled. Then we obtain

$$
\begin{aligned}
P^{T} \tilde{\omega}^{1} P & =\left[\begin{array}{rrrrr}
0 & 0 & 0 & 0 & 0 \\
0 & 0 & 0 & 0 & 0 \\
0 & 0 & 0 & 0 & 0 \\
0 & 0 & 0 & 0 & 1 \\
0 & 0 & 0 & -1 & 0
\end{array}\right], \quad P^{T} \tilde{\omega}^{2} P=\left[\begin{array}{rrrrr}
0 & 0 & 0 & 0 & 0 \\
0 & 0 & 1 & 0 & 0 \\
0 & -1 & 0 & 0 & 0 \\
0 & 0 & 0 & 0 & 0 \\
0 & 0 & 0 & 0 & 0
\end{array}\right], \\
P^{T} \tilde{\omega}^{3} P & =\left[\begin{array}{rrrrr}
0 & 0 & 1 & 0 & 1 \\
0 & 0 & 0 & 0 & 0 \\
-1 & 0 & 0 & 0 & a \\
0 & 0 & 0 & 0 & 0 \\
-1 & 0 & -a & 0 & 0
\end{array}\right], P^{T} \tilde{\omega}^{4} P=\left[\begin{array}{rrrrr}
0 & b & 0 & 1 & 0 \\
-b & 0 & 0 & c & 0 \\
0 & 0 & 0 & 0 & 0 \\
-1 & -c & 0 & 0 & 0 \\
0 & 0 & 0 & 0 & 0
\end{array}\right] .
\end{aligned}
$$

Since

$$
P^{T} \tilde{\omega}_{5} P=\left[\begin{array}{rrrrr}
0 & b & 1 & 1 & 1 \\
-b & 0 & 1 & c & 0 \\
-1 & -1 & 0 & 0 & a \\
-1 & -c & 0 & 0 & 1 \\
-1 & 0 & -a & -1 & 0
\end{array}\right]
$$

has also rank 2 , it must be $a=-b=c=1$. Finally, we have

$$
\omega=\sum_{i=1}^{4} \tilde{\omega}^{i} \tilde{f}^{\pi_{i}}=\left[\begin{array}{rrrrr}
0 & 0 & 0 & 0 & 0 \\
0 & 0 & 0 & 0 & 0 \\
0 & 0 & 0 & 0 & 0 \\
0 & 0 & 0 & 0 & 1 \\
0 & 0 & 0 & -1 & 0
\end{array}\right] \tilde{f}^{\pi_{1}}+\left[\begin{array}{rrrrr}
0 & 0 & 0 & 0 & 0 \\
0 & 0 & 1 & 0 & 0 \\
0 & -1 & 0 & 0 & 0 \\
0 & 0 & 0 & 0 & 0 \\
0 & 0 & 0 & 0 & 0
\end{array}\right] \tilde{f}^{\pi_{2}}
$$




$$
+\left[\begin{array}{rrrrr}
0 & 0 & 1 & 0 & 1 \\
0 & 0 & 0 & 0 & 0 \\
-1 & 0 & 0 & 0 & 1 \\
0 & 0 & 0 & 0 & 0 \\
-1 & 0 & -1 & 0 & 0
\end{array}\right] \tilde{f}^{\pi_{3}}+\left[\begin{array}{rrrrr}
0 & -1 & 0 & 1 & 0 \\
1 & 0 & 0 & 1 & 0 \\
0 & 0 & 0 & 0 & 0 \\
-1 & -1 & 0 & 0 & 0 \\
0 & 0 & 0 & 0 & 0
\end{array}\right] \tilde{f}^{\pi_{4}}
$$

and the following multiplication table:

$$
\begin{aligned}
{\left[f_{4}, f_{5}\right] } & =\tilde{f}^{\pi_{1}}, \\
{\left[f_{2}, f_{3}\right] } & =\tilde{f}^{\pi_{2}}, \\
{\left[f_{1}, f_{3}\right] } & =\left[f_{1}, f_{5}\right]=\left[f_{3}, f_{5}\right]=\tilde{f}^{\pi_{3}}, \\
-\left[f_{1}, f_{2}\right] & =\left[f_{1}, f_{4}\right]=\left[f_{2}, f_{4}\right]=\tilde{f}^{\pi_{4}}, \\
{\left[f_{2}, f_{5}\right] } & =\left[f_{3}, f_{4}\right]=0 .
\end{aligned}
$$

The normal form is given in Sec. 6 (see Eq. (43)).

Case (2). Next, consider the generic case, where two solutions $x^{1}$ and $x^{2}$ are complex conjugate and $x^{3}, x^{4}$, and $x^{5}$ are real and $\operatorname{Re}\left(x^{1}\right), \operatorname{Im}\left(x^{1}\right), x^{3}$, $x^{4}$ and $x^{5}, 4$ by 4 linearly independent as points of $\mathbb{R}^{4}$. Then we can choose $\lambda_{1}, \ldots, \lambda_{5} \neq 0$ such that

$$
\lambda_{1} \operatorname{Re}\left(x^{1}\right)+\lambda_{2} \operatorname{Im}\left(x^{1}\right)+\sum_{i=3}^{4} \lambda_{i} x^{i}=\lambda_{5} x^{5} .
$$

With this choice, $\tilde{\omega}^{1}=\omega\left(\lambda_{1} \operatorname{Re}\left(x^{1}\right)\right), \tilde{\omega}^{2}=\omega\left(\lambda_{2} \operatorname{Im}\left(x^{1}\right)\right)$, and $\tilde{\omega}^{i}=\omega\left(\lambda_{i} x^{i}\right)$ for $i=3,4,5$ are such that

Note that

$$
\sum_{i=1}^{4} \tilde{\omega}^{i}=\tilde{\omega}^{5}
$$

$$
\tilde{\omega}_{1}=\operatorname{Re}\left(\omega\left(\lambda_{1} x^{1}\right)\right), \quad \tilde{\omega}_{2}=\operatorname{Im}\left(\omega\left(\lambda_{2} x^{2}\right)\right) .
$$

Now $\omega\left(\lambda_{1} x^{1}\right)$ has rank 2 and, if $v_{1}, v_{2}$, and $v_{3}$ are three independent vectors of the kernel, then the conjugate vectors $\bar{v}_{1}, \bar{v}_{2}$, and $\bar{v}_{3}$ are independent vectors of the kernel of $\omega\left(\lambda_{2} x^{2}\right)$.

Note that for every $\alpha_{1}, \alpha_{2}, \alpha_{2} \in \mathbb{C}^{3}$,

$$
\omega\left(\lambda_{1} x^{1}\right)\left(\sum \alpha_{i} v_{i}\right)=\omega\left(\lambda_{2} x^{2}\right) \overline{\left(\sum \alpha_{i} v_{i}\right)}=0 .
$$

On the other hand, there exist $\alpha_{i}, i=1,2,3$, such that

$$
\begin{gathered}
v=\sum \alpha_{i} v_{i}=\sum\left(\operatorname{Re}\left(\alpha_{i}\right) \operatorname{Re}\left(v_{i}\right)-\operatorname{Im}\left(\alpha_{i}\right) \operatorname{Im}\left(v_{i}\right)\right) \\
+\imath \sum\left(\operatorname{Re}\left(\alpha_{i}\right) \operatorname{Im}\left(v_{i}\right)+\operatorname{Im}\left(\alpha_{i}\right) \operatorname{Re}\left(v_{i}\right)\right)
\end{gathered}
$$

is a vector with real coefficients, i.e.,

$$
\sum\left(\operatorname{Re}\left(\alpha_{i}\right) \operatorname{Im}\left(v_{i}\right)+\operatorname{Im}\left(\alpha_{i}\right) \operatorname{Re}\left(v_{i}\right)\right)=0 .
$$


Therefore, there exists $v \in \mathbb{R}^{5}$ such that

$$
\omega\left(\lambda_{1} x^{1}\right) v=\omega\left(\lambda_{2} x^{2}\right) v=0
$$

and, in particular, $\tilde{\omega}^{1} v=\tilde{\omega}^{2} v=0$. Then we take $v_{2}, v_{3}$, and $v_{1}=v$ as the basis for the kernel of $\omega\left(\lambda_{1} x^{1}\right)$.

Let $V_{1}$ be the space generated by $\left\{v_{1}, \operatorname{Re} v_{2}, \operatorname{Re} v_{3}\right\}$ and $V_{3}$ and $V_{4}$ be the kernels of $\tilde{\omega}^{3}$ and $\tilde{\omega}^{4}$, respectively. Then we denote $v_{1 j}=V_{1} \cap V_{j}$ for $j=3,4$, and $\hat{v}_{1 j}$ are the vectors such that

$$
\omega\left(\lambda_{1} x^{1}\right)\left(v_{1 j}+\imath \hat{v}_{1 j}\right)=0 .
$$

Finally, if we set $P=\left(\begin{array}{lllll}v_{1} & v_{13} & v_{14} & \hat{v}_{13} & \hat{v}_{14}\end{array}\right)$, we obtain

$$
\begin{aligned}
& \operatorname{Re}\left(\omega\left(\lambda_{1} x^{1}\right)\right) v_{1 j}=\operatorname{Im}\left(\omega\left(\lambda_{1} x^{1}\right)\right) \hat{v}_{1 j}, \\
& \operatorname{Re}\left(\omega\left(\lambda_{1} x^{1}\right)\right) \hat{v}_{1 j}=-\operatorname{Im}\left(\omega\left(\lambda_{1} x^{1}\right)\right) v_{1 j},
\end{aligned}
$$

which implies

$$
\begin{aligned}
\hat{v}_{1 j}^{T} \operatorname{Re}\left(\omega\left(\lambda_{1} x^{1}\right)\right) v_{1 j} & =\hat{v}_{1 j}^{T} \operatorname{Im}\left(\omega\left(\lambda_{1} x^{1}\right)\right) \hat{v}_{1 j}=0, \\
\hat{v}_{1 i}^{T} \operatorname{Im}\left(\omega\left(\lambda_{1} x^{1}\right)\right) v_{1 j} & =v_{1 i}^{T} \operatorname{Re}\left(\omega\left(\lambda_{1} x^{1}\right)\right) v_{1 j} \\
& =v_{1 i}^{T} \operatorname{Im}\left(\omega\left(\lambda_{1} x^{1}\right)\right) \hat{v}_{1 j}=-\hat{v}_{1 i}^{T} \operatorname{Re}\left(\omega\left(\lambda_{1} x^{1}\right)\right) \hat{v}_{1 j}, \\
\hat{v}_{1 i}^{T} \operatorname{Im}\left(\omega\left(\lambda_{1} x^{1}\right)\right) \hat{v}_{1 j} & =v_{1 i}^{T} \operatorname{Re}\left(\omega\left(\lambda_{1} x^{1}\right)\right) \hat{v}_{1 j} \\
& =-v_{1 i}^{T} \operatorname{Im}\left(\omega\left(\lambda_{1} x^{1}\right)\right) v_{1 j}=\hat{v}_{1 i}^{T} \operatorname{Re}\left(\omega\left(\lambda_{1} x^{1}\right)\right) v_{1 j} .
\end{aligned}
$$

Therefore,

$$
\begin{aligned}
P^{T} \operatorname{Re}\left(\omega\left(\lambda_{1} x^{1}\right)\right) P & =\left[\begin{array}{rrrrr}
0 & 0 & 0 & 0 & 0 \\
0 & 0 & a & 0 & b \\
0 & -a & 0 & -b & 0 \\
0 & 0 & b & 0 & -a \\
0 & -b & 0 & a & 0
\end{array}\right], \\
P^{T} \operatorname{Im}\left(\omega\left(\lambda_{1} x^{1}\right)\right) P & =\left[\begin{array}{rrrrr}
0 & 0 & 0 & 0 & 0 \\
0 & 0 & -b & 0 & a \\
0 & b & 0 & -a & 0 \\
0 & 0 & a & 0 & b \\
0 & -a & 0 & -b & 0
\end{array}\right] .
\end{aligned}
$$

Similarly, we obtain a form for $\operatorname{Re}\left(\omega\left(\lambda_{2} x^{2}\right)\right)$ and $\operatorname{Im}\left(\omega\left(\lambda_{2} x^{2}\right)\right)$. Moreover, by a suitable choice of the lengths of the columns of $P$, we obtain

$$
P^{T} \tilde{\omega}^{1} P=P^{T} \operatorname{Re}\left(\omega\left(\lambda_{1} x^{1}\right)\right) P=\left[\begin{array}{rrrrr}
0 & 0 & 0 & 0 & 0 \\
0 & 0 & 1 & 0 & 1 \\
0 & -1 & 0 & -1 & 0 \\
0 & 0 & 1 & 0 & -1 \\
0 & -1 & 0 & 1 & 0
\end{array}\right],
$$




$$
\begin{aligned}
& P^{T} \tilde{\omega}^{2} P=P^{T} \operatorname{Im}\left(\omega\left(\lambda_{2} x^{2}\right)\right) P=\left[\begin{array}{rrrrr}
0 & 0 & 0 & 0 & 0 \\
0 & 0 & -1 & 0 & 1 \\
0 & 1 & 0 & -1 & 0 \\
0 & 0 & 1 & 0 & 1 \\
0 & -1 & 0 & -1 & 0
\end{array}\right], \\
& P^{T}\left(\tilde{\omega}^{3}\right) P=\left[\begin{array}{rrrrr}
0 & 0 & 1 & a_{1} & a_{2} \\
0 & 0 & 0 & 0 & 0 \\
-1 & 0 & 0 & a_{3} & a_{4} \\
-a_{1} & 0 & -a_{3} & 0 & a_{5} \\
-a_{2} & 0 & -a_{4} & -a_{5} & 0
\end{array}\right] \\
& P^{T}\left(\tilde{\omega}^{4}\right) P=\left[\begin{array}{rrrrr}
0 & b_{1} & 0 & b_{2} & b_{3} \\
-b_{1} & 0 & 0 & b_{4} & b_{5} \\
0 & 0 & 0 & 0 & 0 \\
-b_{2} & -b_{4} & 0 & 0 & b_{6} \\
-b_{3} & -b_{5} & 0 & -b_{6} & 0
\end{array}\right] \text {. }
\end{aligned}
$$

Since $\tilde{\omega}^{3}, \tilde{\omega}^{4}$, and $\tilde{\omega}^{5}=\sum_{i} \tilde{\omega}^{i}$ have rank 2 , we have $a_{5}=a_{1} a_{4}-a_{2} a_{3}$, $b_{1} b_{6}=b_{2} b_{5}-b_{3} b_{4}$, and $d_{i}\left(\tilde{\omega}^{5}\right)=0$ for all $i=1, \ldots, 5$, i.e.,

$$
b_{1}=-\frac{b_{2}}{a_{2}}, \quad b_{4}=-\frac{\left(a_{3}-2\right) b_{2}}{a_{2}}, \quad b_{5}=-\frac{2 a_{2}+a_{4} b_{2}}{a_{2}} .
$$

Now we set for all $i \leq j, \tilde{v}_{i j}=\left(P^{T} \tilde{\omega}^{i} P\right) \wedge\left(P^{T} \tilde{\omega}^{j} P\right)$. In particular, we have $\tilde{v}^{11}=\tilde{v}^{22}=[2,0,0,0,0]$ and $\tilde{v}^{33}=\tilde{v}^{44}=\tilde{v}^{12}=[0,0,0,0,0]$.

By choosing $P_{1}=\left[\tilde{v}_{13} \tilde{v}_{14} \tilde{v}_{23} \tilde{v}_{24} \tilde{v}_{34}\right]$ (and suitably rescaling it), we obtain

$$
\begin{aligned}
& \left(P P_{1}\right)^{T} \tilde{\omega}^{1}\left(P P_{1}\right)=\left[\begin{array}{rrrrr}
0 & \frac{1}{2} & 0 & -\frac{1}{2} & 0 \\
-\frac{1}{2} & 0 & \frac{1}{2} & 0 & 0 \\
0 & -\frac{1}{2} & 0 & -\frac{1}{2} & 1 \\
\frac{1}{2} & 0 & \frac{1}{2} & 0 & -1 \\
0 & 0 & -1 & 1 & 0
\end{array}\right], \\
& \left(P P_{1}\right)^{T} \tilde{\omega}^{2}\left(P P_{1}\right)=\left[\begin{array}{rrrrr}
0 & \frac{1}{2} & 0 & \frac{1}{2} & -1 \\
-\frac{1}{2} & 0 & -\frac{1}{2} & 0 & 1 \\
0 & \frac{1}{2} & 0 & -\frac{1}{2} & 0 \\
-\frac{1}{2} & 0 & \frac{1}{2} & 0 & 0 \\
1 & -1 & 0 & 0 & 0
\end{array}\right], \\
& \left(P P_{1}\right)^{T} \tilde{\omega}^{3}\left(P P_{1}\right)=\left[\begin{array}{rrrrr}
0 & 0 & 0 & 0 & 0 \\
0 & 0 & 0 & -1 & 0 \\
0 & 0 & 0 & 0 & 0 \\
0 & 1 & 0 & 0 & 0 \\
0 & 0 & 0 & 0 & 0
\end{array}\right],
\end{aligned}
$$




$$
\left(P P_{1}\right)^{T} \tilde{\omega}^{4}\left(P P_{1}\right)=\left[\begin{array}{rrrrr}
0 & 0 & 1 & 0 & 0 \\
0 & 0 & 0 & 0 & 0 \\
-1 & 0 & 0 & 0 & 0 \\
0 & 0 & 0 & 0 & 0 \\
0 & 0 & 0 & 0 & 0
\end{array}\right] .
$$

Finally, Eq. (2) gives the following multiplication table:

$$
\begin{aligned}
{\left[f_{3}, f_{5}\right] } & =-\left[f_{4}, f_{5}\right]=f^{\pi_{1}}, \\
-\left[f_{1}, f_{5}\right] & =\left[f_{2}, f_{5}\right]=f^{\pi_{2}}, \\
-\left[f_{2}, f_{4}\right] & =f^{\pi_{3}}, \\
{\left[f_{1}, f_{3}\right] } & =f^{\pi_{4}}, \\
{\left[f_{1}, f_{2}\right] } & =-\left[f_{3}, f_{4}\right]=\frac{1}{2}\left(f^{\pi_{1}}+f^{\pi_{2}}\right), \\
{\left[f_{1}, f_{4}\right] } & =-\left[f_{2}, f_{3}\right]=\frac{1}{2}\left(-f^{\pi_{1}}+f^{\pi_{2}}\right) .
\end{aligned}
$$

The normal form is given in Sec. 6 (see Eq. (44)).

Case (3). Finally, we consider the case of two distinct pairs of complex conjugate solutions and only one real solution. We denote these solutions by $x^{1}, x^{2}=\bar{x}^{1} x^{3}, x^{4}=\bar{x}^{2}$, and $x_{5} \in \mathbb{R}^{4}$. Moreover, generically, there exist $\lambda_{1}, \ldots, \lambda_{5} \neq 0$ such that

$$
\lambda_{1} \operatorname{Re}\left(x^{1}\right)+\lambda_{2} \operatorname{Im}\left(x^{1}\right)+\lambda_{3} \operatorname{Re}\left(x^{3}\right)+\lambda_{4} \operatorname{Im}\left(x^{3}\right)=\lambda_{5} x^{5},
$$

and hence, by denoting $y_{1}=\lambda_{1}+\imath \lambda_{2}, y_{2}=\bar{y}_{1}, y_{3}=\lambda_{3}+\imath \lambda_{4}, y_{4}=\bar{y}_{3}$, and $y_{5}=\lambda_{5}$, we have

$$
\operatorname{Re}\left(\bar{y}_{1} x^{1}\right)+\operatorname{Re}\left(\bar{y}_{3} x^{3}\right)=y_{5} x^{5} .
$$

Therefore,

$$
\tilde{\omega}^{1}+\tilde{\omega}^{3}=\tilde{\omega}^{5}
$$

where

$$
\begin{aligned}
& \tilde{\omega}^{1}=\operatorname{Re}\left(\omega\left(\overline{y_{1}} x^{1}\right)\right), \quad \tilde{\omega}^{3}=\operatorname{Re}\left(\omega\left(\overline{y_{3}} x^{3}\right)\right),
\end{aligned}
$$

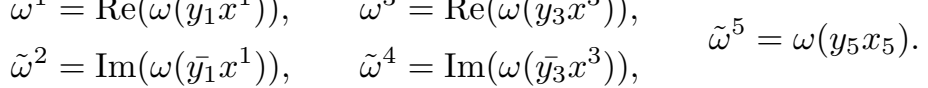

Moreover, $\tilde{\omega}^{1}+\imath \tilde{\omega}^{2}$ and $\tilde{\omega}^{3}+\imath \tilde{\omega}^{4}$ are two complex, rank-2, antisymmetric matrices.

By the same arguments as for the previous case, there exist $v_{1}, v_{2} \in \mathbb{R}^{5}$ such that $\tilde{\omega}^{1} v_{1}=\tilde{\omega}^{2} v_{1}=0$ and $\tilde{\omega}_{3} v_{2}=\tilde{\omega}_{4} v_{2}=0$. Complete $v_{1}, v_{2}$ to a basis of $\mathbb{R}^{5}$. Then, in these coordinates, we can write

$$
\begin{aligned}
\tilde{\omega}^{i}=\left[\begin{array}{c|c}
0 & 0 \\
\hline 0 & \left(\tilde{\omega}^{i}\right)_{22}
\end{array}\right], & i=1,2, \\
\tilde{\omega}^{i}=\left[\begin{array}{c|c}
\left(\tilde{\omega}^{i}\right)_{11} & 0 \\
\hline 0 & 0
\end{array}\right], & i=3,4,
\end{aligned}
$$


where $\left(\tilde{\omega}^{1}\right)_{22},\left(\tilde{\omega}^{2}\right)_{22},\left(\tilde{\omega}^{3}\right)_{11}$, and $\left(\tilde{\omega}^{4}\right)_{11}$ are some antisymmetric $(4 \times 4)$ matrices with determinant 1 . Indeed, since $\tilde{\omega}^{1}+\imath \tilde{\omega}^{2}$ has rank 2 , we can write

$$
0=\left(\tilde{\omega}^{1}+\imath \tilde{\omega}^{2}\right) \wedge\left(\tilde{\omega}^{1}+\imath \tilde{\omega}^{2}\right)=\left(\tilde{\omega}^{1} \wedge \tilde{\omega}^{1}-\tilde{\omega}^{2} \wedge \tilde{\omega}^{2}\right)+2 \imath\left(\tilde{\omega}^{1} \wedge \tilde{\omega}^{2}\right),
$$

which implies that

$$
\tilde{\omega}^{1} \wedge \tilde{\omega}^{2}=0, \quad \tilde{\omega}^{1} \wedge \tilde{\omega}^{1}=\tilde{\omega}^{2} \wedge \tilde{\omega}^{2} .
$$

Hence from the second of Eqs. $(20), \sqrt{\operatorname{det}\left(\left(\tilde{\omega}^{1}\right)_{22}\right)}=\sqrt{\operatorname{det}\left(\left(\tilde{\omega}^{2}\right)_{22}\right)}$. The same relation holds for $\left(\tilde{\omega}^{3}\right)_{11}$ and $\left(\tilde{\omega}^{4}\right)_{11}$.

Now recall that $\tilde{\omega}^{5}=\tilde{\omega}^{1}+\tilde{\omega}^{3}$ is a real rank-2 antisymmetric matrix. Then

$$
0=\tilde{\omega}^{5} \wedge \tilde{\omega}^{5}=\tilde{\omega}^{1} \wedge \tilde{\omega}^{1}+\tilde{\omega}^{3} \wedge \tilde{\omega}^{3}+2 \tilde{\omega}^{1} \wedge \tilde{\omega}^{3},
$$

and hence

$$
\tilde{\omega}^{1} \wedge \tilde{\omega}^{3}=-\frac{1}{2}\left(\tilde{\omega}^{1} \wedge \tilde{\omega}^{1}+\tilde{\omega}^{3} \wedge \tilde{\omega}^{3}\right) .
$$

Finally, denoting $v_{i j}=\tilde{\omega}^{i} \wedge \tilde{\omega}^{j}$ for all $i \leq j$, we have that $v_{11}=v_{22}$, $v_{33}=v_{44}, v_{12}=0, v_{34}=0$ (by Eqs. (20)), and $2 v_{13}+v_{11}+v_{22}=0$ (by Eq. (21)). Then the suitably rescaled matrix $P=\left[\begin{array}{lllll}v_{11} & v_{14} & v_{23} & v_{24} & v_{33}\end{array}\right]$ transforms $\tilde{\omega}^{i}$ as follows:

$$
\begin{aligned}
P^{T} \tilde{\omega}^{1} P= & {\left[\begin{array}{rrrrr}
0 & 0 & 0 & 0 & 0 \\
0 & 0 & -1 & 0 & 0 \\
0 & 1 & 0 & 0 & 0 \\
0 & 0 & 0 & 0 & -1 \\
0 & 0 & 0 & 1 & 0
\end{array}\right], P^{T} \tilde{\omega}^{2} P=\left[\begin{array}{rrrrr}
0 & 0 & 0 & 0 & 0 \\
0 & 0 & 0 & 0 & 1 \\
0 & 0 & 0 & 1 & 0 \\
0 & 0 & -1 & 0 & 0 \\
0 & -1 & 0 & 0 & 0
\end{array}\right], } \\
P^{T} \tilde{\omega}^{3} P= & \left.\begin{array}{rrrrr}
0 & 0 & 0 & 1 & 0 \\
0 & 0 & 1 & 0 & 0 \\
0 & -1 & 0 & 0 & 0 \\
-1 & 0 & 0 & 0 & 0 \\
0 & 0 & 0 & 0 & 0
\end{array}\right], P^{T} \tilde{\omega}^{4} P=\left[\begin{array}{rrrrr}
0 & 0 & -1 & 0 & 0 \\
0 & 0 & 0 & 1 & 0 \\
1 & 0 & 0 & 0 & 0 \\
0 & -1 & 0 & 0 & 0 \\
0 & 0 & 0 & 0 & 0
\end{array}\right] .
\end{aligned}
$$

Finally, we have the following multiplication table:

$$
\begin{gathered}
-\left[f_{4}, f_{5}\right]=f^{\pi_{1}}, \quad\left[f_{2}, f_{5}\right]=\left[f_{3}, f_{4}\right]=f^{\pi_{2}}, \quad\left[f_{1}, f_{4}\right]=f^{\pi_{3}}, \\
-\left[f_{1}, f_{3}\right]=\left[f_{2}, f_{4}\right]=f^{\pi_{4}}, \quad\left[f_{2}, f_{3}\right]=-f^{\pi_{1}}+f^{\pi_{3}}, \\
{\left[f_{1}, f_{2}\right]=\left[f_{1}, f_{5}\right]=\left[f_{3}, f_{5}\right]=0 .}
\end{gathered}
$$

The normal form is given in Sec. 6 (see Eq. (45)).

\section{The CASEs Where $r=3$}

We only have to consider the cases
(i) $d=2$ and $m=1$,
(ii) $d=3$ and $m=1$,
(iii) $d=3$ and $m=7$. 
First we observe that (i) corresponds to the Engel algebra: the growth vector is $(2,3,4)$. It is known that there is only one isomorphism class for this case. For completeness, we give its normal form in Sec. 6 (see Eq. (46)). For (ii) and (iii), the following propositions allow us to reduce the analysis of (iii) to that of (ii).

Proposition 11. Any $E \in \operatorname{Gr}_{m}\left(\mathcal{L}_{3}^{3}\right)$ can be identified with an $m$ dimensional subspace of the space $\Gamma(3)$ of $(3 \times 3)$-matrices $\gamma$ such that $\operatorname{trace}(C \gamma)=0$, where

$$
C=\left[\begin{array}{rrr}
0 & 0 & 1 \\
0 & -1 & 0 \\
1 & 0 & 0
\end{array}\right]
$$

Proof. Fix generators $f_{1}, f_{2}$, and $f_{3}$ for $\mathcal{L}_{3}^{1}$ and let $f^{\pi_{h}}$ be generators of $E \in \operatorname{Gr}_{m}\left(\mathcal{L}_{3}^{3}\right)$. Then we can write

$$
\left[f_{i},\left[f_{j}, f_{k}\right]\right]=\sum_{h=1}^{m} \gamma_{i j k}^{h} f^{\pi_{h}} .
$$

Note that, since

$$
\left[f_{1},\left[f_{2}, f_{3}\right]=\left[f_{2},\left[f_{1}, f_{3}\right]\right]-\left[f_{3},\left[f_{1}, f_{2}\right]\right],\right.
$$

the relation $\gamma_{123}^{h}=\gamma_{213}^{h}-\gamma_{312}^{h}$ holds. Denoting

$$
\gamma^{h}=\left[\begin{array}{ccc}
\gamma_{112}^{h} & \gamma_{212}^{h} & \gamma_{312}^{h} \\
\gamma_{113}^{h} & \gamma_{213}^{h} & \gamma_{313}^{h} \\
\gamma_{123}^{h} & \gamma_{223}^{h} & \gamma_{323}^{h}
\end{array}\right],
$$

we have that trace $\left(C \gamma^{h}\right)=0$ for all $h=1, \ldots, m$. Moreover, if we choose a different set of generators for $E$, i.e.,

$$
f^{\pi_{h}}=\sum_{i=1}^{m} x_{h i} \tilde{f}^{\pi_{i}},
$$

then

$$
\left[f_{i},\left[f_{j}, f_{h}\right]\right]=\sum_{i=1}^{m} \tilde{\gamma}^{i} \tilde{f}^{\pi_{i}}
$$

where

$$
\tilde{\gamma}^{i}=\sum_{h=1}^{m} x_{h i} \gamma^{h}
$$

and

$$
\operatorname{trace}\left(C \tilde{\gamma}^{i}\right)=\sum_{h} x_{h i} \operatorname{trace}\left(C \gamma^{h}\right)=0 .
$$

Then $E$ can be described by an $m$-dimensional subspace in $\Gamma(3)$ generated by $\gamma^{h}, h=1, \ldots, m$. 
Proposition 12. If $r=3$, then the bidimension $\left(d, \ell_{d}^{r-1}+1\right)$ is rigid if and only if the dual bidimension $\left(d, \ell_{d}^{r}-1\right)$ is rigid.

Proof. Now in $\Gamma(3)$, we define the bilinear symmetric product

$$
\Gamma(3) \times \Gamma(3) \rightarrow \mathbb{R}
$$

by $(\gamma, \eta) \mapsto \operatorname{trace}\left(\gamma \eta^{T}\right)$. Then if $E$ is generated by $\gamma^{h}, h=1, \ldots, m$, we define $E^{\perp}$ to be the set of $\gamma$ such that $\left(\gamma, \gamma^{h}\right)=0$ for all $h=1, \ldots, m$.

Now we consider the mappings

$$
\begin{array}{ll}
\Psi: \operatorname{GL}\left(\mathbb{R}^{d}\right) \rightarrow \operatorname{Gr}_{m}\left(\mathcal{L}_{3}^{3}\right), & V \mapsto \Phi(V)(E), \\
\tilde{\Psi}: \operatorname{GL}\left(\mathbb{R}^{d}\right) \rightarrow \operatorname{Gr}_{l_{d}(3)-m}\left(\mathcal{L}_{3}^{3}\right), & V \mapsto \Phi(V)\left(E^{\perp}\right) .
\end{array}
$$

We show that for all $V \in \mathrm{GL}\left(\mathbb{R}^{d}\right), \tilde{\Psi}\left(V^{-T}\right)=(\Psi(V))^{\perp}$, so that a one-to-one correspondence between the image of $\Psi$ and that of $\tilde{\Psi}$ is established and the proposition is proved.

The induced action of $\Phi(V)$ on $\gamma \in \Gamma(3)$ is calculated as follows:

$$
\begin{aligned}
\Phi(V)\left[f_{i},\left[f_{j}, f_{k}\right]\right] & =\left[\sum_{l=1}^{3} v_{i l} f_{l},\left[\sum_{r=1}^{3} v_{j r} f_{r}, \sum_{s=1}^{3} v_{k s} f_{s}\right]\right] \\
& =\sum_{l, s, r=1}^{3} v_{i l} v_{j r} v_{k s}\left[f_{l},\left[f_{r}, f_{s}\right]\right] \\
& =\sum_{l=1}^{3} v_{i l}\left(\sum_{s, r=1}^{3} v_{j r} v_{k s}\left[f_{l},\left[f_{r}, f_{s}\right]\right]\right) \\
& =\sum_{l=1}^{3} v_{i l}\left(\sum_{r<s}\left(v_{j r} v_{k s}-v_{j s} v_{k r}\right)\left[f_{l},\left[f_{r}, f_{s}\right]\right]\right),
\end{aligned}
$$

from which we obtain

Note that

$$
\Phi(V) \gamma^{h}=C V^{-T} C^{T} \gamma^{h} V^{T}, \quad h=1, \ldots, m .
$$

$$
\operatorname{trace}\left(V^{-T} C^{T} \gamma V^{T}\right)=\operatorname{trace}\left(V^{T} V^{-T} C^{T} \gamma\right)=\operatorname{trace}\left(C^{T} \gamma\right)
$$

Therefore,

for all $V \in \mathrm{GL}\left(\mathbb{R}^{3}\right)$. Moreover,

$$
\Phi(V): \Gamma(3) \mapsto \Gamma(3)
$$

$$
\begin{aligned}
\left(\Phi(V) \gamma, \Phi\left(V^{-T}\right) \eta\right) & =\operatorname{trace}\left(\left(C V^{-T} C^{T} \gamma V^{T}\right)\left(C V C^{T} \eta V^{-1}\right)^{T}\right) \\
& =\operatorname{trace}\left(\left(C V^{-T} C^{T} \gamma V^{T}\right)\left(V^{-T} \eta^{T} C V^{T} C^{T}\right)\right) \\
& =\operatorname{trace}\left(\left(C V^{T} C^{T}\right)\left(C V^{-T} C^{T}\right) \gamma \eta^{T}\right) \\
& =\operatorname{trace}\left(\gamma \eta^{T}\right)=0
\end{aligned}
$$

for all $\gamma \in E$ and $\eta \in E^{\perp}$. 
Next, we analyze the case of $m=1$.

Let $\tilde{\Phi}(V): C^{T} \Gamma(3) \rightarrow C^{T} \Gamma(3)$, where $\tilde{\Phi}(V)=C^{T} \circ \Phi\left(V^{T}\right) \circ C$, i.e.,

$$
\tilde{\Phi}(V)\left(C^{T} \gamma\right)=C^{T} C V^{-1} C^{T} \gamma V=V^{-1} C^{T} \gamma V .
$$

The dimensions of the orbit of $\tilde{\Phi}$ and that of $\Phi$ coincide. We have the following assertion.

Lemma 2. The codimension of any orbit of $\tilde{\Phi}$ in $\Gamma(3)$ is greater that or equal to 2 .

Proof. The characteristic polynomial of $C \gamma$ is an invariant of the action of $\tilde{\Phi}$. Indeed,

$$
p(\lambda)=\operatorname{det}\left(V^{-1} C^{T} \gamma V-\lambda I_{3}\right)=\operatorname{det}\left(C^{T} \gamma-\lambda I_{3}\right) .
$$

Since trace $\left(C^{T} \gamma\right)=0$, we have that $p(\lambda)$ is defined by 2 coefficients and the codimension of any orbit of $\tilde{\Phi}$ is no less than 2 .

Proposition 12 and Lemma 2 immediately imply the following assertion.

Proposition 13. The bidimensions $(3,7)$ and $(3,13)$ are not rigid.

\section{The CASES Where $r=4$}

For $r=4$ we only should consider the cases where $d=2$ and $m=1,2$. For $d=2$ and $r=4$, we have $\ell_{2}(4)=3$ brackets of degree 4 which are linearly independent with respect to the Jacobi identity: $\left[f_{1},\left[f_{1},\left[f_{1}, f_{2}\right]\right]\right]$, $\left[f_{2},\left[f_{1},\left[f_{1}, f_{2}\right]\right]\right]$, and $\left[f_{2},\left[f_{2},\left[f_{1}, f_{2}\right]\right]\right]$.

Now let $E \subset \operatorname{Gr}_{m}\left(\mathcal{L}_{2}^{4}\right)$ and $f^{\pi_{h}}, h=1, \ldots, m$, be generators of $E$. Then $E$ can be identified with an $m$-dimensional space of $(2 \times 2)$-symmetric matrices.

Indeed, for all $l, s=1,2$, we can write

$$
\left[f_{l},\left[f_{s},\left[f_{1}, f_{2}\right]\right]\right]=\sum_{h=1}^{m} q_{l s}^{h} f^{\pi_{h}} .
$$

Let $Q^{h}$ be the matrix with coefficients $q_{l s}^{h}$. Since

$$
\lambda_{1}\left[f_{1},\left[f_{1},\left[f_{1}, f_{2}\right]\right]\right]+\lambda_{2}\left[f_{2},\left[f_{2},\left[f_{1}, f_{2}\right]\right]\right]=\left[f_{2},\left[f_{1},\left[f_{1}, f_{2}\right]\right]\right],
$$

we have that $Q^{h}$ are symmetric of order 2. Moreover, if $f^{\pi_{h}}=\sum_{i=1}^{m} x_{h i} \tilde{f}^{\pi_{i}}$, then

$$
\left[f_{l},\left[f_{s},\left[f_{1}, f_{2}\right]\right]\right]=\sum_{i=1}^{m} \tilde{q}_{l s}^{i} \tilde{f}^{\pi_{i}},
$$

where $\tilde{Q}^{i}=\sum_{h=1}^{m} x_{h i} Q^{h}$. 
Next, we compute the induced action of $\Phi(V), V \in \mathbb{R}^{2}$, on the space $\mathcal{S}_{m}(2)$ of symmetric matrices of order 2 corresponding to $E$. From

$$
\begin{aligned}
\Phi(V)\left[f_{l},\left[f_{s},\left[f_{i}, f_{j}\right]\right]\right] & =\left[\sum_{r} v_{l r} f_{r},\left[\sum_{m} v_{s m} f_{m}, \operatorname{det}(V)\left[f_{1}, f_{2}\right]\right]\right] \\
& =\operatorname{det}(V)\left[\sum_{r} v_{l r} f_{r},\left[\sum_{m} v_{s m} f_{m},\left[f_{1}, f_{2}\right]\right]\right] \\
& =\operatorname{det}(V) \sum_{r m} v_{l r} v_{s m}\left[f_{r},\left[f_{m},\left[f_{1}, f_{2}\right]\right]\right]
\end{aligned}
$$

we obtain $\Phi(V) Q^{h}=\operatorname{det}(V) V Q^{h} V^{T}$. Therefore, the degree-2 homogeneous polynomial

$$
\operatorname{det}\left(\sum_{h} x_{h} Q^{h}\right)
$$

in the $m$ variables $x_{1}, \ldots, x_{m}$ is invariant under the action $\Phi$ up to a positive scalar multiplier.

For $m=1$ we have the following assertion.

Proposition 14. The bidimension $(2,6)$ is rigid with two isomorphism classes distinguished by the sign of determinant (23).

Proof. For $m=1$, Eq. (23) reduces to $\operatorname{det}(Q)$, whose sign is invariant under the action $\Phi$. A generic symmetric form $Q$ can be either sign-definite or indefinite (corresponding, respectively, to $\operatorname{det}(Q)>0$ or $\operatorname{det}(Q)<0$ ). For each of these cases, we will give the multiplication table thus showing that $(2,6)$ is rigid with two isomorphism classes.

$m=1, Q$ is positive-definite. Assume that $Q$ is positive definite and of the form

$$
Q=\left[\begin{array}{ll}
2 & 1 \\
1 & 2
\end{array}\right]
$$

Then we have the following multiplication table:

$$
\begin{aligned}
& {\left[f_{2},\left[f_{1},\left[f_{1}, f_{2}\right]\right]\right]=[0,1] Q[1,0]^{T} f^{\pi_{1}}=f^{\pi_{1}},} \\
& {\left[f_{1},\left[f_{1},\left[f_{1}, f_{2}\right]\right]\right]=[1,0] Q[1,0]^{T} f^{\pi_{1}}=2 f^{\pi_{1}},} \\
& {\left[f_{2},\left[f_{2},\left[f_{1}, f_{2}\right]\right]\right]=[0,1] Q[0,1]^{T} f^{\pi_{1}}=2 f^{\pi_{1}} .}
\end{aligned}
$$

The normal form is given in Sec. 6 (see Eq. (47)).

$m=1, Q$ is indefinite. Assume that $Q$ is indefinite and has the form

$$
Q=\left[\begin{array}{ll}
0 & 1 \\
1 & 0
\end{array}\right]
$$


Then we have the following multiplication table:

$$
\begin{aligned}
& {\left[f_{1},\left[f_{2},\left[f_{1}, f_{2}\right]\right]\right]=[1,0] Q[0,1]^{T} f^{\pi_{1}}=f^{\pi_{1}},} \\
& {\left[f_{1},\left[f_{1},\left[f_{1}, f_{2}\right]\right]\right]=[1,0] Q[1,0]^{T} f^{\pi_{1}}=0,} \\
& {\left[f_{2},\left[f_{2},\left[f_{1}, f_{2}\right]\right]\right]=[0,1] Q[0,1]^{T} f^{\pi_{1}}=0 .}
\end{aligned}
$$

The normal form is given in Sec. 6 (see Eq. (25)).

For $m=2$, we have the following assertion.

Proposition 15. The bidimension $(2,7)$ is rigid with two isomorphism classes distinguished by the sign of the discriminant of polynomial (23).

Proof. (23) is a homogeneous polynomial of degree 2 in two variables whose coefficients are invariant under the action of $\Phi$. Then the equation

$$
\operatorname{det}\left(x_{1} Q^{1}+x_{2} Q^{2}\right)=0
$$

has two solutions that can be either real or complex conjugate. For each of these cases, we will give the multiplication table thus showing that $(2,7)$ is rigid with two isomorphism classes.

Remark. The sign of polynomial (23) could serve as an extra invariant in the complex case, but a simple analysis shows that this sign is unavoidably negative.

$m=2$, real case. Assume that Eq. (26) has real solutions. Under generic assumptions, we can assume that they are distinct and that there exist $Q^{1}$ and $Q^{2}$ in the linear space $\mathcal{S}_{2}(2)$ of symmetric $(2 \times 2)$-matrices of order two corresponding to $E$, of corank 1 with transversal kernel. By setting $p_{1}$ and $p_{2}$ to be eigenvectors corresponding to the zero eigenvalue of $Q^{2}$ and $Q^{1}$ respectively, we have that $P=\left[p_{1}, p_{2}\right]$, the matrix of columns $p_{1}$ and $p_{2}$, is such that

$$
\tilde{Q}_{1}=P^{T}\left(Q_{1}\right) P=\left[\begin{array}{ll}
1 & 0 \\
0 & 0
\end{array}\right], \quad \tilde{Q}_{2}=P^{T}\left(Q_{2}\right) P=\left[\begin{array}{ll}
0 & 0 \\
0 & 1
\end{array}\right] .
$$

Finally, we obtain the following multiplication table:

$$
\begin{aligned}
& {\left[f_{1},\left[f_{1},\left[f_{1}, f_{2}\right]\right]\right]=[1,0]\left(\tilde{Q}_{1} f^{\pi_{1}}+\tilde{Q}_{2} f^{\pi_{2}}\right)[1,0]^{T}=f^{\pi_{1}},} \\
& {\left[f_{2},\left[f_{2},\left[f_{1}, f_{2}\right]\right]\right]=[0,1]\left(\tilde{Q}_{1} f^{\pi_{1}}+\tilde{Q}_{2} f^{\pi_{2}}\right)[0,1]^{T}=f^{\pi_{2}},} \\
& {\left[f_{2},\left[f_{1},\left[f_{1}, f_{2}\right]\right]\right]=[0,1]\left(\tilde{Q}_{1} f^{\pi_{1}}+\tilde{Q}_{2} f^{\pi_{2}}\right)[1,0]^{T}=0 .}
\end{aligned}
$$

The normal form is given in Sec. 6 (see Eq. (49)).

$m=2$, complex case. If there exists a pair of complex conjugate solutions of Eq. (26) then we can assume that $Q_{1}$ and $Q_{2}$ in $\mathcal{S}_{2}(2)$ are complex conjugate. Now let $p_{1}$ and $p_{2}$ be such that $p=p_{1}+\imath p_{2} \in \operatorname{ker} Q_{1}$, i.e.,

$$
0=\left(\operatorname{Re}\left(Q_{1}\right)+\imath \operatorname{Im}\left(Q_{1}\right)\right) p
$$




$$
=\left(\operatorname{Re}\left(Q_{1}\right) p_{1}-\operatorname{Im}\left(Q_{1}\right) p_{2}\right)+\imath\left(\operatorname{Re}\left(Q_{1}\right) p_{2}+\operatorname{Im}\left(Q_{1}\right) p_{1}\right),
$$

hence, by setting $P=\left[p_{1}, p_{2}\right]$, we obtain

$$
\begin{aligned}
& \operatorname{Re}\left(\tilde{Q}_{1}\right)=P^{T}\left(\operatorname{Re}\left(Q_{1}\right)\right) P=\left[\begin{array}{rr}
1 & 1 \\
1 & -1
\end{array}\right], \\
& \operatorname{Im}\left(\tilde{Q}_{1}\right)=P^{T}\left(\operatorname{Im}\left(Q_{1}\right)\right) P=\left[\begin{array}{rr}
-1 & 1 \\
1 & 1
\end{array}\right] .
\end{aligned}
$$

Denoting

we have that

$$
f^{\pi_{1}}=\frac{1}{2}\left(\tilde{f}^{\pi_{1}}+\imath \tilde{f}^{\pi_{2}}\right), \quad f^{\pi_{2}}=\frac{1}{2}\left(\tilde{f}^{\pi_{1}}-\imath \tilde{f}^{\pi_{2}}\right),
$$

$$
\tilde{Q}_{1} f^{\pi_{1}}+\tilde{Q}_{2} f^{\pi_{2}}=\operatorname{Re}\left(\tilde{Q}_{1}\right) \tilde{f}^{\pi_{1}}-\operatorname{Im}\left(\tilde{Q}_{1}\right) \tilde{f}^{\pi_{2}}
$$

and obtain the following multiplication table:

$$
\begin{aligned}
& {\left[f_{1},\left[f_{1},\left[f_{1}, f_{2}\right]\right]\right]=[1,0]\left(\operatorname{Re}\left(\tilde{Q}_{1}\right) \tilde{f}^{\pi_{1}}-\operatorname{Im}\left(\tilde{Q}_{1}\right) \tilde{f}^{\pi_{2}}\right)[1,0]^{T}=\tilde{f}^{\pi_{1}}+\tilde{f}^{\pi_{2}},} \\
& {\left[f_{2},\left[f_{2},\left[f_{1}, f_{2}\right]\right]\right]=[0,1]\left(\operatorname{Re}\left(\tilde{Q}_{1}\right) \tilde{f}^{\pi_{1}}-\operatorname{Im}\left(\tilde{Q}_{1}\right) \tilde{f}^{\pi_{2}}\right)[0,1]^{T}=-\tilde{f}^{\pi_{1}}-\tilde{f}^{\pi_{2}},} \\
& {\left[f_{2},\left[f_{1},\left[f_{1}, f_{2}\right]\right]\right]=[0,1]\left(\operatorname{Re}\left(\tilde{Q}_{1}\right) \tilde{f}^{\pi_{1}}-\operatorname{Im}\left(\tilde{Q}_{1}\right) \tilde{f}^{\pi_{2}}\right)[1,0]^{T}=\tilde{f}^{\pi_{1}}-\tilde{f}^{\pi_{2}} .}
\end{aligned}
$$

The normal form is given in Sec. 6 (see Eq. (50)).

\section{NORMAL FORMS}

To calculate the normal form of a set of smooth vector fields $\mathcal{F}$, by the known multiplication table, it suffices to apply the Campbell-Hausdorff formula. Indeed, assume that $\mathcal{F}=\left\{f_{1}, \ldots, f_{d}\right\}$ is regular at $q_{0}$ and the Lie algebra Lie $\mathcal{F}$ is $n$-dimensional with the basis $f^{i}, i=1, \ldots, n$. Then the exponential mapping

$$
\Phi: \sum_{i=1}^{n} x_{i} f^{i} \mapsto q_{0} \exp \left(\sum_{i=1}^{n} x_{i} f^{i}\right)
$$

is smoothly invertible in a neighborhood of $0 \in \operatorname{Lie} \mathcal{F}=\mathbb{R}^{n}$ and defines local coordinates in a neighborhood of $q_{0}$.

Let $q \mapsto q e^{t f^{j}}, t \in \mathbb{R}$, be the flow on $M$ generated by the field $f^{j}$. If $q=\Phi\left(\sum_{i=1}^{n} x_{i} f^{i}\right)$, then

$$
\begin{aligned}
q \exp \left(t f^{j}\right) & =q_{0} \exp \left(\sum_{i=1}^{n} x_{i} f^{i}\right) \exp \left(t f^{j}\right) \\
& =q_{0} \exp \ln \left(\exp \left(\sum_{i=1}^{n} x_{i} f^{i}\right) \exp \left(t f^{j}\right)\right)
\end{aligned}
$$




$$
=\Phi\left(\ln \exp \left(\sum_{i=1}^{n} x_{i} f^{i}\right) \exp \left(t f^{j}\right)\right)
$$

where the product under the logarithm is an element of the "abstract" Lie group generated by the Lie algebra $\mathcal{F}$. Hence the coordinate representation of the flow $q \mapsto q e^{t f^{j}}$ is as follows:

$$
\sum_{i=1}^{n} x_{i} f^{i} \mapsto \ln \left(\exp \left(\sum_{i=1}^{n} x_{i} f^{i}\right) \exp \left(t f^{j}\right)\right),
$$

and the coordinate representation of the vector field $f^{j}$ as a vector-valued function of $x=\left(x_{1}, \ldots, x_{n}\right)$ is

$$
\left.x \mapsto \frac{\partial}{\partial t}\right|_{t=0} \ln \left(\exp \left(\sum_{i=1}^{n} x_{i} f^{i}\right) \exp \left(t f^{j}\right)\right) .
$$

By the Campbell-Hausdorff formula we can write

$$
\begin{aligned}
\ln \left(e^{f} e^{t f^{j}}\right) & =f+t f^{j}+\frac{1}{2}\left[f, t f^{j}\right] \\
& +\frac{1}{12}\left(\left[f,\left[f, t f^{j}\right]\right]-\left[t f^{j},\left[f, t f^{j}\right]\right]\right)-\frac{1}{24}\left[f,\left[t f^{j},\left[f, t f^{j}\right]\right]\right]+\ldots,
\end{aligned}
$$

from which

$$
\left.\frac{d}{d t}\right|_{t=0} \ln \left(e^{f} e^{t f^{j}}\right)=f^{j}+\frac{1}{2}\left[f, f^{j}\right]+\frac{1}{12}\left[f,\left[f, f^{j}\right]\right]+\ldots
$$

Note that in Eq. (29), the brackets of order 4 appear only as a $O\left(t^{2}\right)$ term. Hence in Eq. (30), the brackets of order 4 disappear. Substituting in (30) the expression of $f$ yields:

$$
\left.\frac{\partial}{\partial t}\right|_{t=0} \ln \left(e^{f} e^{t f^{j}}\right)=f^{j}+\frac{1}{2} \sum_{i=1}^{n} x_{i}\left[f^{i}, f^{j}\right]+\frac{1}{12} \sum_{i, h=1}^{n} x_{i} x_{h}\left[f^{i},\left[f^{h}, f^{j}\right]\right]+\ldots
$$

and, finally, substituting the expressions for $\left[f^{i}, f^{j}\right]$ and $\left[f^{i},\left[f^{h}, f^{j}\right]\right]$ as in the multiplication tables yields the expression for $f^{j}$ in the coordinates $\partial / \partial x_{i}$ at the point $x$.

Next we give the resulting expressions of $f^{j}$ for each multiplication table. First, we consider the rigid bidimension $(d, d+1)$ corresponding to $r=2$ and $m=1$. From the multiplication table given in (3), we have $f^{i}=f_{i}$ for $i=1, \ldots, d$ and $f^{d+1}=\left[f_{1}, f_{2}\right]$. Then Eq. (31) has the form

$$
\left.\frac{\partial}{\partial t}\right|_{t=0} \ln \left(e^{f} e^{t f_{j}}\right)=f_{j}+\frac{1}{2} \sum_{i=1}^{d} x_{i}\left[f_{i}, f_{j}\right]
$$


and hence, in the coordinates $\partial / \partial x_{j}$, we have

$$
f_{j}= \begin{cases}\frac{\partial}{\partial x_{j}}+\frac{1}{2} x_{j-1} \frac{\partial}{\partial x_{d+1}} & \text { if } j \text { is even } \\ \frac{\partial}{\partial x_{j}}-\frac{1}{2} x_{j+1} \frac{\partial}{\partial x_{d+1}} & \text { if } j \text { is odd }\end{cases}
$$

For the dual case, the multiplication table is given in (4). Choose $f^{j}$, $j=1, \ldots, n$, as follows:

$$
\begin{array}{ll}
f^{i}=f_{i}, & i=1, \ldots, d, \\
f^{d-1+i}=\left[f_{2 i-1}, f_{2 i}\right], & i=2, \ldots \frac{\hat{d}}{2}, \\
f^{i d+\frac{\hat{d}}{2}-l(i)+k}=\left[f_{i}, f_{k}\right], & k>i,
\end{array}
$$

where $l(i)=\frac{5}{4}+\frac{1}{4}(-1)^{i+1}+\frac{1}{2} i^{2}+i$ and $\hat{d}=d$ if $d$ is even or $\hat{d}=d-1$ if $d$ is odd. Then we have the corresponding normal form:

$$
\begin{aligned}
f_{1} & =\frac{\partial}{\partial x_{1}}-\frac{1}{2} x_{2} \sum_{i=2}^{\frac{\hat{d}}{2}} \frac{\partial}{\partial x_{d-1+i}}-\sum_{i=2}^{\frac{\hat{d}}{2}} \frac{1}{2} x_{i} \frac{\partial}{\partial x_{d+\frac{\hat{d}}{2}-l(1)+i}} \\
f_{2} & =\frac{\partial}{\partial x_{2}}+\frac{1}{2} x_{1} \sum_{i=2}^{\frac{\hat{d}}{2}} \frac{\partial}{\partial x_{d-i+1}}-\sum_{i=2}^{\frac{\hat{d}}{2}} \frac{1}{2} x_{i} \frac{\partial}{\partial x_{2 d+\frac{\hat{d}}{2}-l(2)+i}} \\
f_{j} & =\frac{\partial}{\partial x_{j}}+\sum_{i=1}^{j-2} \frac{x_{i}}{2} \frac{\partial}{\partial x_{i d+\frac{\hat{d}}{2}-l(i)+j}}+\frac{x_{j-1}}{2} \frac{\partial}{\partial x_{d-\frac{j}{2}+1}} \text { if } j \text { is even, } \\
& -\sum_{i=j+1}^{d} \frac{x_{i}}{2} \frac{\partial}{\partial x_{j d+\frac{\hat{d}}{2}-l(j)+i}}-\frac{x_{j+1}}{2} \frac{\partial}{\partial x_{d-\frac{j+1}{2}+1}} \\
f_{j} & =\frac{\partial}{\partial x_{j}}+\sum_{i=1}^{j-1} \frac{x_{i}}{2} \frac{\partial x_{i d+\frac{\hat{d}}{2}-l(i)+j}}{d} j \text { is odd. } \\
& -\sum_{i=j+2} \frac{x_{i}}{2} \frac{\partial}{\partial x_{j d+\frac{\hat{d}}{2}-l(j)+i}}
\end{aligned}
$$

Now we consider the bidimensions $(d, d+2)$ corresponding to $r=2$ and $m=2$. We have rigid cases for $d=4,6$, each with two isomorphism classes, and for $d=5,7$ with one isomorphism class. For $d=4$ and the multiplication table as in Eq. (6), by setting

$$
\begin{gathered}
f^{i}=f_{i}, \quad i=1, \ldots, 4 \\
f^{5}=\left[f_{1}, f_{2}\right]=f^{\pi_{1}}, \quad f^{6}=\left[f_{3}, f_{4}\right]=f^{\pi_{2}},
\end{gathered}
$$


we have that the normal form is

$$
\begin{aligned}
& f_{1}=\frac{\partial}{\partial x_{1}}-\frac{x_{2}}{2} \frac{\partial}{\partial x_{5}} \\
& f_{2}=\frac{\partial}{\partial x_{2}}+\frac{x_{1}}{2} \frac{\partial}{\partial x_{5}} \\
& f_{3}=\frac{\partial}{\partial x_{3}}-\frac{x_{4}}{2} \frac{\partial}{\partial x_{6}}, \\
& f_{4}=\frac{\partial}{\partial x_{4}}+\frac{x_{3}}{2} \frac{\partial}{\partial x_{6}} .
\end{aligned}
$$

For $d=4$ and the multiplication table as in Eq. (7), by setting

$$
\begin{gathered}
f^{i}=f_{i}, \quad i=1, \ldots, 4, \\
f^{5}=\left[f_{1}, f_{3}\right]=-\left[f_{2}, f_{4}\right]=f^{\pi_{1}}, \\
f^{6}=\left[f_{1}, f_{4}\right]=\left[f_{2}, f_{3}\right]=f^{\pi_{2}},
\end{gathered}
$$

we have that the normal form is

$$
\begin{aligned}
f_{1} & =\frac{\partial}{\partial x_{1}}-\frac{x_{3}}{2} \frac{\partial}{\partial x_{5}}-\frac{x_{4}}{2} \frac{\partial}{\partial x_{6}} \\
f_{2} & =\frac{\partial}{\partial x_{2}}+\frac{x_{4}}{2} \frac{\partial}{\partial x_{5}}-\frac{x_{3}}{2} \frac{\partial}{\partial x_{6}} \\
f_{3} & =\frac{\partial}{\partial x_{3}}+\frac{x_{1}}{2} \frac{\partial}{\partial x_{5}}+\frac{x_{2}}{2} \frac{\partial}{\partial x_{6}} \\
f_{4} & =\frac{\partial}{\partial x_{4}}-\frac{x_{2}}{2} \frac{\partial}{\partial x_{5}}+\frac{x_{1}}{2} \frac{\partial}{\partial x_{6}} .
\end{aligned}
$$

For $d=5$, considering the multiplication table (??) and setting

$$
\begin{gathered}
f^{i}=f_{i}, \quad i=1, \ldots, 5, \\
f^{6}=\left[f_{1}, f_{2}\right]=\left[f_{3}, f_{5}\right]=f^{\pi_{1}}, \\
f^{7}=\left[f_{3}, f_{4}\right]=\left[f_{1}, f_{5}\right]=f^{\pi_{2}},
\end{gathered}
$$

we obtain

$$
\begin{aligned}
& f_{1}=\frac{\partial}{\partial x_{1}}-\frac{x_{2}}{2} \frac{\partial}{\partial x_{6}}-\frac{x_{5}}{2} \frac{\partial}{\partial x_{7}} \\
& f_{2}=\frac{\partial}{\partial x_{2}}+\frac{x_{1}}{2} \frac{\partial}{\partial x_{6}}, \\
& f_{3}=\frac{\partial}{\partial x_{3}}-\frac{x_{5}}{2} \frac{\partial}{\partial x_{6}}-\frac{x_{4}}{2} \frac{\partial}{\partial x_{7}} \\
& f_{4}=\frac{\partial}{\partial x_{4}}+\frac{x_{3}}{2} \frac{\partial}{\partial x_{7}}, \\
& f_{5}=\frac{\partial}{\partial x_{5}}+\frac{x_{3}}{2} \frac{\partial}{\partial x_{6}}+\frac{x_{1}}{2} \frac{\partial}{\partial x_{7}}
\end{aligned}
$$


For $d=6$ with multiplication table as in (8), by setting

$$
\begin{gathered}
f^{i}=f_{i}, \quad i=1, \ldots, 6, \\
{\left[f_{1}, f_{2}\right]=f^{7}, \quad\left[f_{3}, f_{4}\right]=f^{8},}
\end{gathered}
$$

we have:

$$
\begin{aligned}
f_{1} & =\frac{\partial}{\partial x_{1}}-\frac{1}{2} x_{2} \frac{\partial}{\partial x_{7}}, \\
f_{2} & =\frac{\partial}{\partial x_{2}}+\frac{1}{2} x_{1} \frac{\partial}{\partial x_{7}}, \\
f_{3} & =\frac{\partial}{\partial x_{3}}-\frac{1}{2} x_{4} \frac{\partial}{\partial x_{8}}, \\
f_{4} & =\frac{\partial}{\partial x_{4}}+\frac{1}{2} x_{3} \frac{\partial}{\partial x_{8}}, \\
f_{5} & =\frac{\partial}{\partial x_{5}}+\frac{1}{2} x_{6}\left(\frac{\partial}{\partial x_{7}}-\frac{\partial}{\partial x_{8}}\right), \\
f_{6} & =\frac{\partial}{\partial x_{6}}-\frac{1}{2} x_{5}\left(\frac{\partial}{\partial x_{7}}-\frac{\partial}{\partial x_{8}}\right) .
\end{aligned}
$$

For $d=6$ with multiplication table as in (9) and setting

$$
\begin{gathered}
f^{i}=f_{i}, \quad i=1, \ldots, 6, \\
f^{7}=\left[f_{3}, f_{5}\right]=-\left[f_{4}, f_{6}\right]=f^{\pi_{1}}, \\
f^{8}=\left[f_{3}, f_{6}\right]=\left[f_{4}, f_{5}\right]=f^{\pi_{2}},
\end{gathered}
$$

we have:

$$
\begin{aligned}
f_{1} & =\frac{\partial}{\partial x_{1}}-\frac{1}{2} x_{2}\left(\frac{\partial}{\partial x_{7}}-\frac{\partial}{\partial x_{8}}\right), \\
f_{2} & =\frac{\partial}{\partial x_{2}}+\frac{1}{2} x_{1}\left(\frac{\partial}{\partial x_{7}}-\frac{\partial}{\partial x_{8}}\right), \\
f_{3} & =\frac{\partial}{\partial x_{3}}-\frac{1}{2} x_{5} \frac{\partial}{\partial x_{7}}-\frac{1}{2} x_{6} \frac{\partial}{\partial x_{8}}, \\
f_{4} & =\frac{\partial}{\partial x_{4}}+\frac{1}{2} x_{6} \frac{\partial}{\partial x_{7}}-\frac{1}{2} x_{5} \frac{\partial}{\partial x_{8}}, \\
f_{5} & =\frac{\partial}{\partial x_{5}}+\frac{1}{2} x_{3} \frac{\partial}{\partial x_{7}}+\frac{1}{2} x_{4} \frac{\partial}{\partial x_{8}}, \\
f_{6} & =\frac{\partial}{\partial x_{6}}-\frac{1}{2} x_{4} \frac{\partial}{\partial x_{7}}+\frac{1}{2} x_{3} \frac{\partial}{\partial x_{8}} .
\end{aligned}
$$

Finally, for $d=7$, whose multiplication table is given in (??), by setting

$$
f^{i}=f_{i}, \quad i=1, \ldots, 7,
$$

$$
\begin{aligned}
& f^{8}=\left[f_{1}, f_{2}\right]=\left[f_{3}, f_{7}\right]=\left[f_{5}, f_{7}\right]=f^{\pi_{1}}, \\
& f^{9}=\left[f_{3}, f_{4}\right]=\left[f_{1}, f_{7}\right]=\left[f_{6}, f_{7}\right]=f^{\pi_{2}},
\end{aligned}
$$


we have the following normal form:

$$
\begin{aligned}
f_{1} & =\frac{\partial}{\partial x_{1}}-\frac{1}{2} x_{2} \frac{\partial}{\partial x_{8}}-\frac{1}{2} x_{7} \frac{\partial}{\partial x_{9}}, \\
f_{2} & =\frac{\partial}{\partial x_{2}}+\frac{1}{2} x_{1} \frac{\partial}{\partial x_{8}}, \\
f_{3} & =\frac{\partial}{\partial x_{3}}-\frac{1}{2} x_{7} \frac{\partial}{\partial x_{8}}-\frac{1}{2} x_{4} \frac{\partial}{\partial x_{9}}, \\
f_{4} & =\frac{\partial}{\partial x_{4}}+\frac{1}{2} x_{3} \frac{\partial}{\partial x_{9}}, \\
f_{5} & =\frac{\partial}{\partial x_{5}}+\frac{1}{2}\left(x_{6}-x_{7}\right) \frac{\partial}{\partial x_{8}}-\frac{1}{2} x_{6} \frac{\partial}{\partial x_{9}}, \\
f_{6} & =\frac{\partial}{\partial x_{6}}-\frac{1}{2} x_{5} \frac{\partial}{\partial x_{8}}+\frac{1}{2}\left(x_{5}-x_{7}\right) \frac{\partial}{\partial x_{9}}, \\
f_{7} & =\frac{\partial}{\partial x_{7}}+\frac{1}{2}\left(x_{3}+x_{5}\right) \frac{\partial}{\partial x_{8}}+\frac{1}{2}\left(x_{1}+x_{6}\right) \frac{\partial}{\partial x_{9}} .
\end{aligned}
$$

Next we consider the bidimensions $(d, d+3)$ corresponding to $r=2$ and $m=3$. We have seen that such bidimensions are rigid for $d=4$ and $d=5$ with two isomorphism classes.

For $d=4$ and multiplication table (14), by setting

$$
\begin{gathered}
f^{i}=f_{i}, \quad i=1, \ldots, 4 \\
f^{5}=f^{\pi_{1}}=-\left[f_{1}, f_{2}\right]-f^{\pi_{3}}, \quad f^{6}=f^{\pi_{2}}=\left[f_{3}, f_{4}\right]+f^{\pi_{3}}, \\
f^{7}=\left[f_{1}, f_{3}\right]=\left[f_{2}, f_{4}\right]=f^{\pi_{3}}
\end{gathered}
$$

we obtain:

$$
\begin{aligned}
& f_{1}=\frac{\partial}{\partial x_{1}}+\frac{1}{2} x_{2} \frac{\partial}{\partial x_{5}}+\frac{1}{2}\left(x_{2}-x_{3}\right) \frac{\partial}{\partial x_{7}} \\
& f_{2}=\frac{\partial}{\partial x_{2}}-\frac{1}{2} x_{1} \frac{\partial}{\partial x_{5}}-\frac{1}{2}\left(x_{1}+x_{4}\right) \frac{\partial}{\partial x_{7}} \\
& f_{3}=\frac{\partial}{\partial x_{3}}-\frac{1}{2} x_{4} \frac{\partial}{\partial x_{6}}+\frac{1}{2}\left(x_{1}+x_{4}\right) \frac{\partial}{\partial x_{7}} \\
& f_{4}=\frac{\partial}{\partial x_{4}}+\frac{1}{2} x_{3} \frac{\partial}{\partial x_{6}}+\frac{1}{2}\left(x_{2}-x_{3}\right) \frac{\partial}{\partial x_{7}}
\end{aligned}
$$

For $d=4$ and multiplication table (16), by setting

$$
\begin{gathered}
f^{i}=f_{i}, \quad i=1, \ldots, 4, \\
f^{5}=f^{\pi_{1}}, \quad f^{6}=f^{\pi_{2}}, \\
f^{7}=\left[f_{1}, f_{2}\right]=\left[f_{3}, f_{4}\right]=f^{\pi_{3}},
\end{gathered}
$$


we obtain:

$$
\begin{aligned}
& f_{1}=\frac{\partial}{\partial x_{1}}-\frac{1}{2}\left(x_{3}+x_{4}\right) \frac{\partial}{\partial x_{5}}+\frac{1}{2}\left(x_{3}-x_{4}\right) \frac{\partial}{\partial x_{6}}-\frac{1}{2} x_{2} \frac{\partial}{\partial x_{7}} \\
& f_{2}=\frac{\partial}{\partial x_{2}}-\frac{1}{2}\left(x_{3}-x_{4}\right) \frac{\partial}{\partial x_{5}}-\frac{1}{2}\left(x_{3}+x_{4}\right) \frac{\partial}{\partial x_{6}}+\frac{1}{2} x_{1} \frac{\partial}{\partial x_{7}} \\
& f_{3}=\frac{\partial}{\partial x_{3}}+\frac{1}{2}\left(x_{1}+x_{2}\right) \frac{\partial}{\partial x_{5}}-\frac{1}{2}\left(x_{1}-x_{2}\right) \frac{\partial}{\partial x_{6}}-\frac{1}{2} x_{4} \frac{\partial}{\partial x_{7}} \\
& f_{4}=\frac{\partial}{\partial x_{4}}+\frac{1}{2}\left(x_{1}-x_{2}\right) \frac{\partial}{\partial x_{5}}+\frac{1}{2}\left(x_{1}+x_{2}\right) \frac{\partial}{\partial x_{6}}+\frac{1}{2} x_{3} \frac{\partial}{\partial x_{7}} .
\end{aligned}
$$

For $d=5$ and multiplication table (17), by setting

$$
\begin{gathered}
f^{i}=f_{i}, \quad i=1, \ldots, 5, \\
f^{6}=\left[f_{2}, f_{5}\right]=\left[f_{3}, f_{4}\right]=f^{\pi_{1}}, \\
f^{7}=-\left[f_{1}, f_{4}\right]=-\left[f_{3}, f_{5}\right]=f^{\pi_{2}}, \\
f^{8}=\left[f_{1}, f_{3}\right]=-\left[f_{2}, f_{3}\right]=\mp\left[f_{4}, f_{5}\right]=f^{\pi_{3}},
\end{gathered}
$$

we have:

$$
\begin{aligned}
& f_{1}=\frac{\partial}{\partial x_{1}}+\frac{1}{2} x_{4} \frac{\partial}{\partial x_{7}}-\frac{1}{2} x_{3} \frac{\partial}{\partial x_{8}}, \\
& f_{2}=\frac{\partial}{\partial x_{2}}-\frac{1}{2} x_{5} \frac{\partial}{\partial x_{6}}+\frac{1}{2} x_{3} \frac{\partial}{\partial x_{8}}, \\
& f_{3}=\frac{\partial}{\partial x_{3}}-\frac{1}{2} x_{4} \frac{\partial}{\partial x_{6}}+\frac{1}{2} x_{5} \frac{\partial}{\partial x_{7}}+\frac{1}{2}\left(x_{1}-x_{2}\right) \frac{\partial}{\partial x_{8}}, \\
& f_{4}=\frac{\partial}{\partial x_{4}}+\frac{1}{2} x_{3} \frac{\partial}{\partial x_{6}}-\frac{1}{2} x_{1} \frac{\partial}{\partial x_{7}} \pm \frac{1}{2} x_{5} \frac{\partial}{\partial x_{8}}, \\
& f_{5}=\frac{\partial}{\partial x_{5}}+\frac{1}{2} x_{2} \frac{\partial}{\partial x_{6}}-\frac{1}{2} x_{3} \frac{\partial}{\partial x_{7}} \mp \frac{1}{2} x_{4} \frac{\partial}{\partial x_{8}} .
\end{aligned}
$$

Now we consider the bidimension $(d, d+4)$ corresponding to $r=2$ and $m=4$. This bidimension is rigid with three isomorphism classes for $d=5$. First, we consider the multiplication table as in (18) and set

$$
\begin{gathered}
f^{i}=f_{i}, \quad i=1, \ldots, 5, \\
f^{6}=\left[f_{4}, f_{5}\right]=f^{\pi_{1}}, \quad f^{7}=\left[f_{2}, f_{3}\right]=f^{\pi_{2}}, \\
f^{8}=\left[f_{1}, f_{3}\right]=\left[f_{1}, f_{5}\right]=\left[f_{3}, f_{5}\right]=f^{\pi_{3}}, \\
f^{9}=-\left[f_{1}, f_{2}\right]=\left[f_{1}, f_{4}\right]=\left[f_{2}, f_{4}\right]=f^{\pi_{4}} .
\end{gathered}
$$


The normal form is

$$
\begin{aligned}
f_{1} & =\frac{\partial}{\partial x_{1}}-\frac{1}{2}\left(x_{3}+x_{5}\right) \frac{\partial}{\partial x_{8}}+\frac{1}{2}\left(x_{2}-x_{4}\right) \frac{\partial}{\partial x_{9}} \\
f_{2} & =\frac{\partial}{\partial x_{2}}-\frac{1}{2} x_{3} \frac{\partial}{\partial x_{7}}-\frac{1}{2}\left(x_{1}+x_{4}\right) \frac{\partial}{\partial x_{9}} \\
f_{3} & =\frac{\partial}{\partial x_{3}}+\frac{1}{2} x_{2} \frac{\partial}{\partial x_{7}}+\frac{1}{2}\left(x_{1}-x_{5}\right) \frac{\partial}{\partial x_{8}} \\
f_{4} & =\frac{\partial}{\partial x_{4}}-\frac{1}{2} x_{5} \frac{\partial}{\partial x_{6}}+\frac{1}{2}\left(x_{1}+x_{2}\right) \frac{\partial}{\partial x_{9}} \\
f_{5} & =\frac{\partial}{\partial x_{5}}+\frac{1}{2} x_{4} \frac{\partial}{\partial x_{6}}+\frac{1}{2}\left(x_{1}+x_{3}\right) \frac{\partial}{\partial x_{8}}
\end{aligned}
$$

Next, for the multiplication table as in (19), setting

$$
\begin{gathered}
f^{i}=f_{i}, \quad i=1, \ldots, 5, \\
f^{6}=\left[f_{3}, f_{5}\right]=-\left[f_{4}, f_{5}\right]=f^{\pi_{1}}, \\
f^{7}=-\left[f_{1}, f_{5}\right]=\left[f_{2}, f_{5}\right]=f^{\pi_{2}}, \\
f^{8}=-\left[f_{2}, f_{4}\right]=f^{\pi_{3}}, \quad f^{9}=\left[f_{1}, f_{3}\right]=f^{\pi_{4}},
\end{gathered}
$$

we have the following normal form:

$$
\begin{aligned}
& f_{1}=\frac{\partial}{\partial x_{1}}-\frac{1}{4}\left(x_{2}-x_{4}\right) \frac{\partial}{\partial x_{6}}-\frac{1}{4}\left(x_{2}+x_{4}-2 x_{5}\right) \frac{\partial}{\partial x_{7}}-\frac{1}{2} x_{3} \frac{\partial}{\partial x_{9}}, \\
& f_{2}=\frac{\partial}{\partial x_{2}}+\frac{1}{4}\left(x_{1}-x_{3}\right) \frac{\partial}{\partial x_{6}}+\frac{1}{4}\left(x_{1}+x_{3}-2 x_{5}\right) \frac{\partial}{\partial x_{7}}+\frac{1}{2} x_{4} \frac{\partial}{\partial x_{8}}, \\
& f_{3}=\frac{\partial}{\partial x_{3}}+\frac{1}{4}\left(x_{2}+x_{4}-2 x_{5}\right) \frac{\partial}{\partial x_{6}}-\frac{1}{4}\left(x_{2}-x_{4}\right) \frac{\partial}{\partial x_{7}}+\frac{1}{2} x_{1} \frac{\partial}{\partial x_{9}}, \\
& f_{4}=\frac{\partial}{\partial x_{4}}-\frac{1}{4}\left(x_{1}+x_{3}-2 x_{5}\right) \frac{\partial}{\partial x_{6}}+\frac{1}{4}\left(x_{1}-x_{3}\right) \frac{\partial}{\partial x_{7}}-\frac{1}{2} x_{2} \frac{\partial}{\partial x_{8}}, \\
& f_{5}=\frac{\partial}{\partial x_{5}}+\frac{1}{2}\left(x_{3}-x_{4}\right) \frac{\partial}{\partial x_{6}}-\frac{1}{2}\left(x_{1}-x_{2}\right) \frac{\partial}{\partial x_{7}} .
\end{aligned}
$$

Finally, for the multiplication table as in (22), setting

$$
\begin{gathered}
f^{i}=f_{i}, \quad i=1, \ldots, 5, \\
f^{6}=-\left[f_{4}, f_{5}\right]=f^{\pi_{1}}, \quad f^{7}=\left[f_{2}, f_{5}\right]=\left[f_{3}, f_{4}\right]=f^{\pi_{2}}, \\
f^{8}=\left[f_{1}, f_{4}\right]=f^{\pi_{3}}, \quad f^{9}=-\left[f_{1}, f_{3}\right]=\left[f_{2}, f_{4}\right]=f^{\pi_{4}},
\end{gathered}
$$


we obtain the normal form

$$
\begin{aligned}
& f_{1}=\frac{\partial}{\partial x_{1}}-\frac{1}{2} x_{4} \frac{\partial}{\partial x_{8}}+\frac{1}{2} x_{3} \frac{\partial}{\partial x_{9}}, \\
& f_{2}=\frac{\partial}{\partial x_{2}}-\frac{1}{2} x_{5} \frac{\partial}{\partial x_{7}}-\frac{1}{2} x_{4} \frac{\partial}{\partial x_{9}}+\frac{1}{2} x_{3}\left(\frac{\partial}{\partial x_{6}}-\frac{\partial}{\partial x_{8}}\right), \\
& f_{3}=\frac{\partial}{\partial x_{3}}-\frac{1}{2} x_{4} \frac{\partial}{\partial x_{7}}-\frac{1}{2} x_{1} \frac{\partial}{\partial x_{9}}-\frac{1}{2} x_{2}\left(\frac{\partial}{\partial x_{6}}-\frac{\partial}{\partial x_{8}}\right), \\
& f_{4}=\frac{\partial}{\partial x_{4}}+\frac{1}{2} x_{5} \frac{\partial}{\partial x_{6}}+\frac{1}{2} x_{3} \frac{\partial}{\partial x_{7}}+\frac{1}{2} x_{1} \frac{\partial}{\partial x_{8}}+\frac{1}{2} x_{2} \frac{\partial}{\partial x_{9}} \\
& f_{5}=\frac{\partial}{\partial x_{5}}-\frac{1}{2} x_{4} \frac{\partial}{\partial x_{6}}+\frac{1}{2} x_{2} \frac{\partial}{\partial x_{7}} .
\end{aligned}
$$

For $r=3$, the unique rigid case is the Engel algebra. The multiplication table is given by

$$
\begin{gathered}
f^{i}=f_{i}, \quad i=1,2, \\
f^{3}=\left[f_{1}, f_{2}\right], \quad f^{4}=\left[f_{1}, f^{3}\right]=\left[f_{2}, f^{3}\right] .
\end{gathered}
$$

The normal form of $\mathcal{F}$ is

$$
\begin{aligned}
& f_{1}=\frac{\partial}{\partial x_{1}}-\frac{1}{2} x_{2} \frac{\partial}{\partial x_{3}}-\frac{1}{12}\left(6 x_{3}+x_{1} x_{2}+x_{2}^{2}\right) \frac{\partial}{\partial x_{4}}, \\
& f_{2}=\frac{\partial}{\partial x_{2}}+\frac{1}{2} x_{1} \frac{\partial}{\partial x_{3}}-\frac{1}{12}\left(6 x_{3}-x_{1} x_{2}-x_{1}^{2}\right) \frac{\partial}{\partial x_{4}} .
\end{aligned}
$$

For $r=4$, the unique rigid bidimensions are $(2,6)$ and $(2,7)$; the correspond to $d=2, m=1$ and $d=2, m=2$ respectively. For $n=6,7$, we calculate the brackets $\left[f, f_{j}\right]$ by setting $f^{i}=f_{i}$ for $i=1,2$ and $f^{3}=\left[f_{1}, f_{2}\right]$, $f^{4}=\left[f_{1}, f^{3}\right]$, and $f^{5}=\left[f_{2}, f^{3}\right]$ :

$$
\begin{aligned}
{\left[f, f_{1}\right] } & =\sum_{i=1}^{n} x_{i}\left[f_{i}, f_{1}\right]=+x_{2}\left[f_{2}, f_{1}\right]+x_{3}\left[\left[f_{1}, f_{2}\right], f_{1}\right] \\
& +x_{4}\left[\left[f_{1},\left[f_{1}, f_{2}\right]\right], f_{1}\right]+x_{5}\left[\left[f_{2},\left[f_{1}, f_{2}\right]\right], f_{1}\right] \\
& =-x_{2}\left[f_{1}, f_{2}\right]-x_{3}\left[f_{1},\left[f_{1}, f_{2}\right]\right] \\
& -x_{4}\left[f_{1},\left[f_{1},\left[f_{1}, f_{2}\right]\right]\right]-x_{5}\left[f_{1},\left[f_{2},\left[f_{1}, f_{2}\right]\right]\right], \\
{\left[f, f_{2}\right] } & =\sum_{i=1}^{n} x_{i}\left[f_{i}, f_{2}\right]=+x_{1}\left[f_{1}, f_{2}\right]+x_{3}\left[\left[f_{1}, f_{2}\right], f_{2}\right] \\
& +x_{4}\left[\left[f_{1},\left[f_{1}, f_{2}\right]\right], f_{2}\right]+x_{5}\left[\left[f_{2},\left[f_{1}, f_{2}\right]\right], f_{2}\right] \\
& =+x_{1}\left[f_{1}, f_{2}\right]-x_{3}\left[f_{2},\left[f_{1}, f_{2}\right]\right] \\
& -x_{4}\left[f_{2},\left[f_{1},\left[f_{1}, f_{2}\right]\right]\right]-x_{5}\left[f_{2},\left[f_{2},\left[f_{1}, f_{2}\right]\right]\right], \\
{\left[f,\left[f, f_{1}\right]\right] } & =-x_{2}\left(x_{1}\left[f_{1},\left[f_{1}, f_{2}\right]\right]+x_{2}\left[f_{2},\left[f_{1}, f_{2}\right]\right]\right) \\
& -x_{3}\left(x_{1}\left[f_{1},\left[f_{1},\left[f_{1}, f_{2}\right]\right]\right]+x_{2}\left[f_{2},\left[f_{1},\left[f_{1}, f_{2}\right]\right]\right]\right),
\end{aligned}
$$




$$
\begin{aligned}
{\left[f,\left[f, f_{2}\right]\right] } & =+x_{1}\left(x_{1}\left[f_{1},\left[f_{1}, f_{2}\right]\right]+x_{2}\left[f_{2},\left[f_{1}, f_{2}\right]\right]\right) \\
& -x_{3}\left(x_{1}\left[f_{1},\left[f_{2},\left[f_{1}, f_{2}\right]\right]\right]+x_{2}\left[f_{2},\left[f_{2},\left[f_{1}, f_{2}\right]\right]\right]\right) .
\end{aligned}
$$

Then according to the multiplication table (24) for $m=1$ and setting $f^{6}=\left[f_{2}, f^{4}\right]=f^{\pi_{1}}$, we have:

$$
\begin{aligned}
f_{1} & =\frac{\partial}{\partial x_{1}}-\frac{1}{2}\left(x_{2} \frac{\partial}{\partial x_{3}}+x_{3} \frac{\partial}{\partial x_{4}}+\left(2 x_{4}+x_{5}\right) \frac{\partial}{\partial x_{6}}\right) \\
& -\frac{1}{12}\left(x_{2} x_{1} \frac{\partial}{\partial x_{4}}+x_{2}^{2} \frac{\partial}{\partial x_{5}}+\left(2 x_{3} x_{1}+x_{3} x_{2}\right) \frac{\partial}{\partial x_{6}}\right) \\
& =\frac{\partial}{\partial x_{1}}-\frac{1}{2} x_{2} \frac{\partial}{\partial x_{3}}-\frac{1}{12}\left(6 x_{3}+x_{1} x_{2}\right) \frac{\partial}{\partial x_{4}}-\frac{1}{12} x_{2}^{2} \frac{\partial}{\partial x_{5}} \\
& -\frac{1}{12}\left(12 x_{4}+6 x_{5}+2 x_{1} x_{3}+x_{2} x_{3}\right) \frac{\partial}{\partial x_{6}} \\
f_{2} & =\frac{\partial}{\partial x_{2}}+\frac{1}{2}\left(x_{1} \frac{\partial}{\partial x_{3}}-x_{3} \frac{\partial}{\partial x_{5}}-\left(x_{4}+2 x_{5}\right) \frac{\partial}{\partial x_{6}}\right) \\
& +\frac{1}{12}\left(x_{1}^{2} \frac{\partial}{\partial x_{4}}+x_{1} x_{2} \frac{\partial}{\partial x_{5}}-\left(x_{3} x_{1}+2 x_{3} x_{2}\right) \frac{\partial}{\partial x_{6}}\right) \\
& =\frac{\partial}{\partial x_{2}}+\frac{1}{2} x_{1} \frac{\partial}{\partial x_{3}}+\frac{1}{12} x_{1}^{2} \frac{\partial}{\partial x_{4}}-\frac{1}{12}\left(6 x_{3}-x_{1} x_{2}\right) \frac{\partial}{\partial x_{5}} \\
& -\frac{1}{12}\left(12 x_{5}+6 x_{4}+2 x_{2} x_{3}+x_{1} x_{3}\right) \frac{\partial}{\partial x_{6}} .
\end{aligned}
$$

For the multiplication table (25), setting $f^{6}=\left[f_{1}, f^{5}\right]=f^{\pi_{1}}$, we have instead the following normal form:

$$
\begin{aligned}
f_{1} & =\frac{\partial}{\partial x_{1}}-\frac{1}{2}\left(x_{2} \frac{\partial}{\partial x_{3}}+x_{3} \frac{\partial}{\partial x_{4}}+x_{5} \frac{\partial}{\partial x_{6}}\right) \\
& -\frac{1}{12}\left(x_{2} x_{1} \frac{\partial}{\partial x_{4}}+x_{2}^{2} \frac{\partial}{\partial x_{5}}+x_{3} x_{2} \frac{\partial}{\partial x_{6}}\right) \\
& =\frac{\partial}{\partial x_{1}}-\frac{1}{2} x_{2} \frac{\partial}{\partial x_{3}}-\frac{1}{12}\left(6 x_{3}+x_{1} x_{2}\right) \frac{\partial}{\partial x_{4}} \\
& -\frac{1}{12} x_{2}^{2} \frac{\partial}{\partial x_{5}}-\frac{1}{12}\left(6 x_{5}+x_{2} x_{3}\right) \frac{\partial}{\partial x_{6}}, \\
f_{2} & =\frac{\partial}{\partial x_{2}}+\frac{1}{2}\left(x_{1} \frac{\partial}{\partial x_{3}}-x_{3} \frac{\partial}{\partial x_{5}}-x_{4} \frac{\partial}{\partial x_{6}}\right) \\
& +\frac{1}{12}\left(x_{1}^{2} \frac{\partial}{\partial x_{4}}+x_{1} x_{2} \frac{\partial}{\partial x_{5}}-x_{1} x_{3} \frac{\partial}{\partial x_{6}}\right) \\
& =\frac{\partial}{\partial x_{2}}+\frac{1}{2} x_{1} \frac{\partial}{\partial x_{3}}+\frac{1}{12} x_{1}^{2} \frac{\partial}{\partial x_{4}} \\
& -\frac{1}{12}\left(6 x_{3}-x_{1} x_{2}\right) \frac{\partial}{\partial x_{5}}-\frac{1}{12}\left(6 x_{4}+x_{1} x_{3}\right) \frac{\partial}{\partial x_{6}} .
\end{aligned}
$$


For $m=2$ and the multiplication table (27), setting

$$
f^{6}=\left[f_{1}, f^{4}\right]=f^{\pi_{1}}, \quad f^{7}=\left[f_{2}, f^{5}\right]=f^{\pi_{2}},
$$

we have

$$
\begin{aligned}
f_{1} & =\frac{\partial}{\partial x_{1}}-\frac{1}{2}\left(x_{2} \frac{\partial}{\partial x_{3}}+x_{3} \frac{\partial}{\partial x_{4}}+x_{4} \frac{\partial}{\partial x_{6}}\right) \\
& -\frac{1}{12}\left(x_{2} x_{1} \frac{\partial}{\partial x_{4}}+x_{2}^{2} \frac{\partial}{\partial x_{5}}+x_{3} x_{1} \frac{\partial}{\partial x_{6}}\right) \\
& =\frac{\partial}{\partial x_{1}}-\frac{1}{2} x_{2} \frac{\partial}{\partial x_{3}}-\frac{1}{12}\left(6 x_{3}+x_{1} x_{2}\right) \frac{\partial}{\partial x_{4}} \\
& -\frac{1}{12} x_{2}^{2} \frac{\partial}{\partial x_{5}}-\frac{1}{12}\left(6 x_{4}+x_{1} x_{3}\right) \frac{\partial}{\partial x_{6}}, \\
f_{2} & =\frac{\partial}{\partial x_{2}}+\frac{1}{2}\left(x_{1} \frac{\partial}{\partial x_{3}}-x_{3} \frac{\partial}{\partial x_{5}}-x_{5} \frac{\partial}{\partial x_{7}}\right) \\
& +\frac{1}{12}\left(x_{1}^{2} \frac{\partial}{\partial x_{4}}+x_{1} x_{2} \frac{\partial}{\partial x_{5}}-x_{3} x_{2} \frac{\partial}{\partial x_{7}}\right) \\
& =\frac{\partial}{\partial x_{2}}+\frac{1}{2} x_{1} \frac{\partial}{\partial x_{3}}+\frac{1}{12} x_{1}^{2} \frac{\partial}{\partial x_{4}} \\
& -\frac{1}{12}\left(6 x_{3}-x_{1} x_{2}\right) \frac{\partial}{\partial x_{5}}-\frac{1}{12}\left(6 x_{5}+x_{2} x_{3}\right) \frac{\partial}{\partial x_{7}} .
\end{aligned}
$$

Finally, for the multiplication table (28), setting $f^{6}=f^{\pi_{1}}$ and $f^{7}=f^{\pi_{2}}$, we have:

$$
\begin{gathered}
f_{1}=\frac{\partial}{\partial x_{1}}+\frac{1}{2}\left(-x_{2} \frac{\partial}{\partial x_{3}}-x_{3} \frac{\partial}{\partial x_{4}}-x_{4}\left(\frac{\partial}{\partial x_{6}}+\frac{\partial}{\partial x_{7}}\right)-x_{5}\left(\frac{\partial}{\partial x_{6}}-\frac{\partial}{\partial x_{7}}\right)\right) \\
+\frac{1}{12}\left(-x_{1} x_{2} \frac{\partial}{\partial x_{4}}-x_{2}^{2} \frac{\partial}{\partial x_{5}}-x_{1} x_{3}\left(\frac{\partial}{\partial x_{6}}+\frac{\partial}{\partial x_{7}}\right)-x_{2} x_{3}\left(\frac{\partial}{\partial x_{6}}-\frac{\partial}{\partial x_{7}}\right)\right) \\
=\frac{\partial}{\partial x_{1}}-\frac{1}{2} x_{2} \frac{\partial}{\partial x_{3}}-\frac{1}{12}\left(6 x_{3}+x_{1} x_{2}\right) \frac{\partial}{\partial x_{4}}-\frac{1}{12} x_{2}^{2} \frac{\partial}{\partial x_{5}} \\
-\frac{1}{12}\left(6 x_{4}+6 x_{5}+x_{1} x_{3}+x_{2} x_{3}\right) \frac{\partial}{\partial x_{6}}+\frac{1}{12}\left(-6 x_{4}+6 x_{5}-x_{1} x_{3}+x_{2} x_{3}\right) \frac{\partial}{\partial x_{7}}, \\
f_{2}=\frac{\partial}{\partial x_{2}}+\frac{1}{2}\left(x_{1} \frac{\partial}{\partial x_{3}}-x_{3} \frac{\partial}{\partial x_{5}}-x_{4}\left(\frac{\partial}{\partial x_{6}}-\frac{\partial}{\partial x_{7}}\right)-x_{5}\left(-\frac{\partial}{\partial x_{6}}-\frac{\partial}{\partial x_{7}}\right)\right) \\
+\frac{1}{12}\left(x_{1}^{2} \frac{\partial}{\partial x_{4}}+x_{1} x_{2} \frac{\partial}{\partial x_{5}}-x_{3} x_{1}\left(\frac{\partial}{\partial x_{6}}-\frac{\partial}{\partial x_{7}}\right)-x_{3} x_{2}\left(-\frac{\partial}{\partial x_{6}}-\frac{\partial}{\partial x_{7}}\right)\right) \\
=\frac{\partial}{\partial x_{2}}+\frac{1}{2} x_{1} \frac{\partial}{\partial x_{3}}+\frac{1}{12} x_{1}^{2} \frac{\partial}{\partial x_{4}}-\frac{1}{12}\left(6 x_{3}-x_{1} x_{2}\right) \frac{\partial}{\partial x_{5}} \\
-\frac{1}{12}\left(6 x_{4}-6 x_{5}+x_{1} x_{3}-x_{2} x_{3}\right) \frac{\partial}{\partial x_{6}}+\frac{1}{12}\left(6 x_{4}+6 x_{5}+x_{1} x_{3}+x_{2} x_{3}\right) \frac{\partial}{\partial x_{7}} .
\end{gathered}
$$




\section{REFERENCES}

1. A. A. Agrachev, R. V. Gamkrelidze, and A. V. Sarychev, Local invariants of smooth control systems. Acta Appl. Math. 14 (1989), 191-237.

2. A. Agrachev and A. Marigo, Nonholonomic tangent spaces: Intrinsic construction and rigid dimensions. Electronic Res. Announcements AMS 9 (2003), 111-120.

3. A. A. Agrachev and A. V. Sarychev, Filtrations of a Lie algebra of vector fields and nilpotent approximation of control systems. Sov. Math. Dokl. 36 (1988), 104-108.

4. A. Bellaiche, The tangent space in sub-Riemannian geometry. In: SubRiemannian geometry. Progr. Math. 144 (1996), 1-78.

5. R. M. Bianchini and G. Stefani, Graded approximations and controllability along a trajectory. SIAM J. Control Optim. 28 (1990), 903-924.

6. W.-L. Chow, Über Systeme von linearen partiellen Differentialgleichungen erster Ordnung. Math. Ann. 117 (1940-41), 98-105.

7. F. Gantmakher, The theory of matrices, v.2. Chelsea Publ. Comp. (1959), 276p.

8. Algebraische Reduktion der Scharen bilinearer Formen, Sitzungsber. Akademie Berlin (1890), 763-776.

9. P. K. Rashevsky, About connecting two points of a completely nonholonomic space by admissible curve. Uch. Zap. Pedag. Inst. Libknechta 2 (1938), 83-94.

10. L. M. Rothschild and E. M. Stein, Hypoelliptic differential operators and nilpotent groups. Acta Math. 137 (1976), 247-320.

11. A. M. Vershik and V. Ya. Gershkovich, Nonholonomic dynamic systems. Geometry of Distributions and Variational Problems. Springer Verlag 16.

(Received March 02 2004, received in revised form March 21 2005)

Authors' addresses:

A. Agrachev

SISSA, Trieste, Italy

E-mail: agrachev@sissa.it

A. Marigo

Università di Roma "La Sapienza", Italy

E-mail: marigo@mat.uniroma1.it 